The Scaling of Seismic Energy With Moment: Simple Models Compared With Observations

W. R. Walter, K. Mayeda, R. Gok, A. Hofstetter

January 11, 2006

American Geophysical Union Monograph 170 
This document was prepared as an account of work sponsored by an agency of the United States Government. Neither the United States Government nor the University of California nor any of their employees, makes any warranty, express or implied, or assumes any legal liability or responsibility for the accuracy, completeness, or usefulness of any information, apparatus, product, or process disclosed, or represents that its use would not infringe privately owned rights. Reference herein to any specific commercial product, process, or service by trade name, trademark, manufacturer, or otherwise, does not necessarily constitute or imply its endorsement, recommendation, or favoring by the United States Government or the University of California. The views and opinions of authors expressed herein do not necessarily state or reflect those of the United States Government or the University of California, and shall not be used for advertising or product endorsement purposes. 


\title{
The Scaling of Seismic Energy With Moment: Simple Models Compared With Observations
}

\author{
William R. Walter, Kevin Mayeda and Rengin Gok \\ Earth Sciences Division, Lawrence Livermore National Laboratory, Livermore, California \\ Abraham Hofstetter \\ Seismology, Geophysical Institute of Israel, Lod, Israel
}

The scaling between small and large earthquakes remains an outstanding issue For many years the predominant hypothesis has been that the rupture process is self-similar, leading to predictions that source parameters such as apparent stress are the same for all earthquakes, except as affected by initial conditions. As digital broadband data has become widely available, a number of recent empirical studies have challenged self-similarity, though the evidence remains mixed. Using simple point source models in the time and frequency domains, we review the predicted scaling behavior of earthquake energy and other source parameters, under self-similar and non-self-similar assumptions. The models make clear that self-similar scaling leads to some testable hypotheses, including the constancy of apparent stress and the spectral shape under a particular frequency transformation, regardless of the true (and perhaps unknown) source time function. We also review some of the problems posed by measurement errors in determining seismic energy and the limited magnitude ranges of events within given studies to answering the scaling question. We use the 1999 Hector Mine California earthquake sequence in California to apply several different techniques to address the scaling question. Using regional direct and coda wave types, and examining scaling using both spectral determination of seismic energy and frequency domain spectral shape scaling, we show the Hector Mine sequence shows evidence of non-self-similar scaling. Finally we briefly look at the types of models that appear to be consistent with the earthquake apparent stress behavior that has been observed to date.

\section{INTRODUCTION}

How do initially small ruptures grow into large earthquakes? The answer to this question gets to heart of our scientific knowledge of earthquakes. It also directly impacts our ability to use the more ubiquitous small earthquakes to forecast the effects of larger, more damaging events. One approach to answering this question is to first understand the relationship of fundamental, measurable, physical parameters between small and large events. Is a magnitude 8 earthquake simply a scaled up magnitude 3 event, or do basic quantities such as apparent stress (or scaled energy), stress drop, and rupture velocity vary with size? For many years the prevailing idea has been that these parameters are independent of earthquake size, im- 
plying that the rupture process itself is scale invariant (e.g. Aki 1967). With the advent of widespread broadband digital seismometers over the past 15 years or so, this selfsimilar model is undergoing more rigorous testing than ever before. Indeed quite a few recent studies have found evidence for departures from self-similarity, although the geophysical community remains divided on the issue. This overview paper sets out to review the most general models of earthquake self-similarity versus non-self similar models, with their implications for observable parameters such as radiated energy, their compatibility with published studies and makes some suggestions for future work to resolve the issue.

\section{MOMENT AND ENERGY}

We focus on two key earthquake observable parameters the seismic moment $\left(M_{0}\right)$ and radiated energy $\left(E_{R}\right)$. Seismic moment is a static measure of earthquake size. It is a quantitative measure the amount of earth movement during an event and is independent of the details of the rupture time history. It is defined as the product of the shear modulus $\mu$, the average slip $\bar{D}$ and the fault area A:

$$
M_{o}=\mu \bar{D} A
$$

The radiated energy is a dynamic measure of earthquake size. It explicitly depends upon details of the rupture process. Slip on a fault that occurs very slowly, such as creep, may radiate very little energy compared with a felt earthquake, yet both could have the same moment. Radiated energy can also be defined as the product of fault area and average slip when multiplied by an apparent stress $\sigma_{a}$ (e.g. Wyss 1970):

$$
E_{R}=\sigma_{a} \bar{D} A
$$

Apparent stress is the fraction of the effective applied stress on the fault that does not go to frictional heating, the rupture of atomic bonds and other local energy consumptive processes. It is tied to the energy in the seismic waves. To better understand the dynamic behavior of earthquakes with changes in size, we take the ratio of radiated energy and moment, basically normalizing the energy radiated to form the scaled energy $\tilde{e}=E_{R} / M_{o}$. We see that the scaled energy is proportional to the apparent stress $\left(\sigma_{a}=\mu \tilde{e}\right)$ through the shear modulus, and so both will have the same scaling behavior. 
A number of recent studies have looked at the behavior of scaled energy or apparent stress with earthquake size and come to opposite conclusions. In Table 1 we list on the left-hand side a number of very persuasive studies that find that scaled energy increases with earthquake size. On the right-hand side we list a number of equally compelling studies that conclude that scaled energy is constant (with scatter) as earthquake size changes. The question of how scaled energy behaves with size gets at the physics of earthquakes. If earthquakes have constant scaled energy regardless of size it implies that similar physics applies and a magnitude 8 is simply a magnitude 3 earthquake that continues to grow. If scaled energy increases with size it implies that larger events radiate more seismic energy per unit fault slip than small ones. In other words larger earthquakes are more efficient radiators of seismic energy, which implies the rupture dynamics of small and large events differ. This may also imply that there are clues to the eventual size of large earthquakes even at the very beginning of rupture, a topic of much current discussion (e.g. Olson and Allen, 2005; Abercrombie, 2005).

Table 1. Some examples of papers examining observational evidence for earthquake energy scaling.

\begin{tabular}{|l|l|}
\hline $\mathrm{E}_{\mathrm{R}} / \mathrm{M}_{\mathrm{o}}$ increasing with size & \multicolumn{1}{|c|}{ Constant $\mathrm{E}_{\mathrm{R}} / \mathrm{M}_{\mathrm{o}}$} \\
\hline Kanamori et al. $(1993)$ & Kanamori and Anderson \\
Abercrombie (1995) & $(1975)$ \\
Mayeda and Walter (1996) & Singh and Ordaz (1994) \\
Izutani and Kanamori & Choy and Boatwright \\
$(2001)$ & $(1995)$ \\
Prejean and Ellsworth & McGarr (1999) \\
(2001) & Ide and Beroza (2001) \\
Richardson and Jordan & Ide et al. $(2003)$ \\
(2002) & Matsuzawa et al (2004) \\
Mori et al. (2003) & Prieto et al. (2004) \\
Stork and Ito (2004) & Yamada et al. (2005) \\
Mayeda et al (2005) & Jin and Fukuyama (2005) \\
Izutani (2005) & \\
Takahashi et al. (2005) & \\
\hline
\end{tabular}

The reasons for the conflicting conclusions of the studies listed in Table 1 lie almost entirely in the estimation of radiated seismic energy. Seismic moment determination using waveform modeling is fairly mature. It is unusual for different investigators to have seismic moments determinations for the same event differ by more than a factor of two (e.g. Ritsema and Lay, 1995; Pasyanos et al 1996). In contrast, energy estimation for the same events using different methods, such as regional versus teleseismic data techniques, can differ by factors much larger than two (e.g. Singh and Ordaz 1994, Mayeda and Walter, 1996; PerzCampos et al. 2003). 
Several factors are responsible for this uncertainty in the amount of energy radiated for each event. First, the majority of seismic energy radiated at the source is in S-waves and concentrated in frequencies within about a factor of about ten of the so-called corner frequency. Energy estimates that do not directly sample S-waves or cannot sample this whole frequency band must make corrections that are subject to sizeable uncertainties. Second, amplitudes at these frequencies are subject to significant path and site effects including geometrical spreading, attenuation, and amplification/deamplification due to the surficial layers. Third, the source contains directivity and other inhomogeneities in the radiation pattern that may be difficult to account for and can bias the results if there is insufficient sampling of the focal sphere to average out these effects (e.g., Favereau and Archuleta, 2003).

In recent years a variety of studies have looked at ways to improve the error surrounding the estimate of energy, or to more directly look at the scaling behavior of earthquakes. Before looking at observational results, it will be helpful to understand the role of moment, energy and scaled energy in the context of basic physical models of earthquakes.

\section{SIMPLE EARTHQUAKE MODELS}

\subsection{General Properties}

The simplest and most general model of an earthquake source is the far-field point-source model in the time and frequency domains. Far-field indicates that we are more than several wavelengths away from the source for all frequencies considered. In the point source approximation we look only at one moment tensor source with a single source time function radiating from a point. Such a source is applicable to the azimuthally averaged radiation rather than any specific seismogram. No matter how complex the source we can always find frequencies at which the approximation is valid, and those frequencies in fact scale with earthquake size as well. We believe much physical insight can be gained by understanding the behavior of such models. We return at the end of this section to discuss some of the limitations of this viewpoint.

Let us define the moment time function, $M_{o}(t)$ as the change in moment due to earthquake slip along a fault as defined in equation 1 . In practice we may not know the specific shape of the moment time function, and we can use an arbitrary shape such as shown in Figure 1 without loss 
of generality. We define the earthquake to occur over a duration $\tau$ with no significant movement on the fault before and afterwards. Taking the first derivative in time we get the moment rate time function $\dot{M}_{o}(t)$, which can be shown to be proportional to the far field seismic displacement waves (e.g. Aki and Richards, 1980). Let us define the peak of the moment rate time function as $P$. The moment is proportional the area under this curve and therefore proportional the product of the peak times the duration: $P \tau$. If the fault does not reverse directions during slip, the moment rate function will be all positively valued. In any case it has the same duration $\tau$, which varies with earthquake size.

In the frequency domain the moment rate spectrum is the Fourier transform of the moment rate function, which is proportional to the displacement spectrum. In its simplest form it has a basic shape on a log-log plot as shown in Figure 1. As the frequency goes to zero the displacement spectral amplitude becomes constant and proportional to the moment. If the fault does not reverse directions during slip this zero frequency asymptote will be the maximum in the displacement spectrum. At high frequencies the spectral amplitude must decay with increasing frequency at a rate greater than $\mathrm{f}^{1.5}$ in order to keep the energy bounded (e.g. Walter and Brune 1993). The simplest spectral shape that matches these criteria has the form of two asymptotes, one constant at low frequencies and one decaying as $\mathrm{f}^{\psi}$ where $\psi>1.5$ at high frequencies (e.g. Aki, 1967; Brune 1970). The intersection of these two asymptotes is commonly called the corner frequency and is proportional to the reciprocal of the earthquake duration.

The seismic radiated energy is related to the velocity seismograms. Taking another time derivative of the moment rate function we get the moment acceleration function $\ddot{M}_{o}(t)$, which is proportional to the far-field velocity. The seismic energy is proportional to the integral of the velocity squared (e.g. Kanamori et al., 1993). In the frequency domain the velocity spectrum is equal to the displacement spectrum divided by the circular frequency $2 \pi f$, giving a peak amplitude near the corner frequency. This implies the spectral energy density peak is centered around the corner frequency. Given that the energy is proportional to the integral of the velocity squared spectra we can see that most of the energy is within frequencies about an order of magnitude above and below the corner frequency. Studies that cannot sample such a range frequencies when making energy measurements are subject to sizable uncertainties (e.g. Ide and Beroza, 2001). Durations and corner frequencies change with earthquake size so that scaling studies need to operate over a very large range of frequencies in- 
deed.

One additional important point is that for earthquakes the majority of the seismic energy is radiated as S-waves. To quantify this we need to make a few further assumptions. Treating the earthquake point source as a double couple (or shear slip on a planar surface) and assuming that the $\mathrm{P}$ wave source spectrum has a similar but scaled shape as the $\mathrm{S}$-wave spectrum, the ratio of energy in $\mathrm{S}$ waves to $\mathrm{P}$ waves, $q$, was given by Boatwright and Fletcher, (1984) as:

$$
q=\frac{3}{2}\left(\frac{\alpha}{\beta}\right)^{5}\left(\frac{f_{c S}}{f_{c P}}\right)^{3} .
$$

For crustal earthquakes we can assume the relationship between the P-wave velocity $\alpha$ and the S-wave velocity $\beta$ is approximately Poissonian or $\alpha=\sqrt{3} \beta$. The ratio of the $\mathrm{P}$-wave corner frequency $f_{c P}$ to the $\mathrm{S}$-wave corner frequency $f_{c S}$ depends upon the details of the fault rupture. However, we can bound the values between 1 and $\sqrt{3}$ using physical principles (e.g. Boatwright and Fletcher, 1984; Walter and Brune, 1993). If the rupture duration is long compared to the fault dimension over the wave velocities then the observed corner frequency is determined by the duration and the corner frequencies are equal. If the fault ruptures instantaneously the corner frequencies are proportional to the wave velocities over the fault dimension, so the corner frequency ratio is the ratio of the wave velocities or about $\sqrt{3} \sim 1.73$. Putting these end member values into equation 3 we see that $q$ varies from 4.5 to 23.4 as the corner frequency ratio varies from 1.73 to 1 . In practice we expect the corner frequency ratio to fall between these extremes, but in all cases the S-wave energy dominates. This implies that energy estimates using P-waves alone must make sizable corrections to get absolute values. Comparative energy studies using P-waves alone can be done, but then the question of the corner frequency variation between events becomes part of the problem, and in general this is a poorly known quantity.

\subsection{Self-Similar Scaling}

Consider an arbitrary earthquake fault surface with dimension $L$ as shown in Figure 2. Under self-similar scaling we can scale the earthquake up (or down) in size by a factor $x$ and keep all physical properties constant. In this case observable parameters will scale as the fault dimension $L$ scales. So for example the fault area will scale as $x^{2}$. The average fault slip for a crack in an elastic media is proportional to the dimension $L$ through the stress drop divided by 
the rigidity (e.g. Eshelby, 1957) and so will scale as $x$. The moment is defined in equation 1 . Substituting for the fault slip and area we see it is proportional to the dimension cubed and scales as $x^{3}$. The duration is proportional to the average slip divided by the rupture velocity, $V$, which is proportional to length $L$ and so scales as $x$. We can use the simple model in Figure 1 to get at the radiated energy, which is proportional to $P^{2} / \tau$. In the time domain the moment rate time function has a peak amplitude $P$ and duration $\tau$. The area under the curve is the moment, which is proportional to $P \tau$. Since we have shown that $M_{o}$ scales as $x^{3}$ and $\tau$ as $x$ then $P$ must scale as $x^{2}$. From this scaling we can see that the energy scales as $x^{3}$, the same as the moment, and thus the scaled energy $\tilde{e}=E_{R} / M_{o}$ will be constant in this model.

In the frequency domain the spectral scaling of the selfsimilar model is shown in Figure 3. The low frequency level scales as the moment and the corner frequency scales as $f^{3}$. To see this we can write the relationship between moment, duration and corner frequency:

$$
M_{o} \sim \Delta \sigma L^{3} \sim \Delta \sigma V^{3} \tau^{3} \sim\left(\Delta \sigma V^{3}\right) f_{c}^{-3}
$$

Implicit in the self-similar model are that the stress drop and rupture velocity are constant, although equation 4 shows that only the product in the parentheses needs to be constant for the spectral scaling shown (see also Kanamori and Rivera, 2004). An interesting property of the selfsimilar model that follows from equation 4 is that any spectral model that is invariant under $f^{3}$ scaling has constant scaled energy. This is true for the common self-similar models of Aki (1967) and Brune (1970) but is also true for any particular shape or high frequency falloff that follow such scaling. This means that if one imagines sliding the spectral curves along a line of $f^{3}$ the spectra will all overlie each other. Prieto et al. (2004) have cleverly exploited this property to investigate the scaling behavior of scaled energy, a point we will return to later.

\subsection{Non-Self-Similar Scaling}

Here we consider two types of non-self-similar models as alternatives. One in which the spectral shape remains invariant but under a different frequency scaling, and one in which the shape itself changes with size. 
variant under a different frequency scaling than $f^{3}$ it can be shown that the scaled energy is no longer constant. For example in figure 4 we show spectra that are invariant un$\operatorname{der} f^{4}$ frequency scaling. Following the notation of $\mathrm{Ka}$ namori and Rivera (2004) we define the scaling between moment and corner frequency as:

$$
M_{o} \sim f_{c}^{-(3+\varepsilon)}
$$

where $\varepsilon$ (epsilon) represents the deviation from selfsimilarity and is usually thought to be a small positive number. In Figure $4 \varepsilon=1$. From equations 4 and 5 we can show the scaling behavior of stress drop and rupture velocity in terms of corner frequency and $\varepsilon$ and then rewrite it in terms of moment from equation 5 :

$$
\left(\Delta \sigma V^{3}\right) \sim f_{c}^{-\varepsilon} \sim M_{o}^{\varepsilon /(3+\varepsilon)}
$$

From equation (6) we can see one way in which non-selfsimilarity can occur, when stress drop and/or rupture velocity increase with moment. If the rupture velocity changes with earthquake size this implies some scaling in rupture physics. If the stress drop scales with size it can be interpreted as an indication that the initial conditions vary with larger events occurring in regions with higher stress drop (e.g. Beeler, 2003). However, then one would need a physical explanation of why the faults fail at different stress levels in different regions.

The scaled energy can also be written in terms of a deviation from cube root scaling in terms of epsilon. Perhaps the easiest way to see this is to write the scaled energy in terms of the simple model shown in Figure 1

$$
\tilde{e}=\frac{E_{R}}{M_{o}} \sim \frac{P^{2} / \tau}{P \tau} \sim \frac{P}{\tau^{2}} \sim \frac{M_{o}}{\tau^{3}} \sim M_{o} f_{c}^{3}
$$

where we have made use of $\tau \sim f_{c}^{-1}$. From equation 7 we can see that scaled energy will be constant when the peak displacement scales as the duration squared in the time domain. For the frequency domain we can see again that scaled energy is constant when the moment scales as the cube root of the corner frequency, as already shown in equation (4). If the corner frequency scaling follows the non-self-similar form of equation (5) then the scaled energy will vary with moment, which we can determine by substituting equation (5) into equation (7) to obtain: 


$$
\tilde{e} \sim M_{o}^{\varepsilon /(3+\varepsilon)}
$$

For the example shown in Figure 4, where $\varepsilon=1$, then $\tilde{e} \sim M_{o}^{1 / 4}$. We can see that in the frequency domain, if we can find a value of epsilon where the source spectra all overlie each other then we can determine the scaling of $\tilde{e}$ from directly from equation (8). Rivera and Kanamori (2004) showed that the particular shape that gives a minimum scaled energy follows the form of equation (8) in the case of a circular fault. However equation (8) as derived here shows that this is a general relation for any fault model where the moment rate function (in the time domain) or the displacement spectrum (in the frequency domain) have the same shape, such that equation (7) is true. This means that the spectral shape could be quite complex, with multiple corner frequencies and still be invariant under scaling of the form of equation (5). In those cases one should think of $f_{c}$ as a characteristic frequency, related to the reciprocal of duration and not necessarily a corner frequency.

An alternative way of thinking about non-self-similar models is in terms of efficiency. If we consider a shear fault, we can define an efficiency $\eta$, which is the fraction of the available elastic energy that goes in seismic radiation. In Figure 5 we show an example of a spectra with the same moment but changing efficiency from a circular shear fault. Decreasing efficiency has the effect of decreasing the corner frequency. If the efficiency increases with earthquake size it could have the effect of shifting the corner frequency beyond that expected from self-similar models leading to values of $\varepsilon$ in equation 5 greater than zero.

There are a variety of very interesting theoretical models that argue that earthquake efficiency increases as earthquake size or slip increases. For example, if the dynamic or sliding friction were to decrease as earthquake size increased it could cause this effect. There has been a lot of experimental work studying sliding friction, as well as numerical simulation studies investigating earthquake rupture dynamics, looking at the idea of velocity or slip-weakening friction laws. In such laws as sliding begins it causes the friction to decrease, leading to further sliding and a sustainable earthquake. It is known that large earthquakes occur with lower than expected driving stress (e.g., Heaton, 1990). Many ideas have been proposed for reducing dynamic friction through some kind of slip weakening process, including shear melting (e.g., Jefferies, 1942; Kanamori and Heaton, 2000), acoustic fluidization (Melosh, 1979), rough fault sliding induced normal stress reduction (e.g., Brune et al., 1993), fluid pressurization (e.g., Sibson, 1973) and elastohydrodynamic lubrication (Brodsky and 
Kanamori, 2001). Observational constraints on energy release per moment would help us understand and distinguish between these models.

We compare the self-similar and non-self similar models from figure 3 and 4 in Figure 6. For this comparison we have chosen to have them match up at $\mathrm{M}_{\mathrm{w}}=5$. This plot shows how difficult it can be to distinguish between these two models. For small magnitude events, like $\mathrm{M}_{\mathrm{w}}=2$ there are clear differences at high frequencies $(f>10 \mathrm{~Hz})$, but observations at these frequencies are subject to very strong site and path corrections that trade-off with source estimation. At large magnitudes such as $\mathrm{M}_{\mathrm{w}}=8$ there are significant amplitude differences at all frequencies, however such large magnitude events are rare. We return to the issue of matching observations to models in the next section.

3.3.2 Changing spectral shape. An alternate way in which earthquake spectral models could be non-self-similar would be if their spectral (and corresponding time domain) shapes changed systematically with moment. For example in Figure 7 we show a hypothetical case in which for small magnitudes the spectra have a single corner frequency, but at larger magnitudes a second corner frequency is introduced. In this case the frequency band of intermediate falloff grows with earthquake size. Such intermediate falloff has been observed in some large earthquakes (e.g. Mayeda and Walter, 1996). There are a number of possible reasons for the appearance of an additional corner frequency as earthquake size increases. One possibility is if earthquakes change from equal-dimensional growth to uni-dimensional growth as the fault dimension approaches the depth of the seismogenic region (e.g. Romanowicz and Ruff, 2002) and this introduces a second corner frequency. Another possibility is if larger events have partial stress drop (e.g. Brune, 1970) which introduces a second corner frequency. The idea that small earthquakes are simple and large ones are complex has a heuristic appeal to many seismologists. However it is incompatible with self-similarity, which argues that all earthquakes are equally complex, just the frequency or time scale of the complexities varies as shown in Figures 1 and 3. If large earthquakes are really more complex (after scaling) than small ones, earthquakes are not self-similar. 
There are many other possible shape changes with size that would also violate self-similarity. For example the high frequency falloff could vary with size or the complexity of the time or frequency domain shape itself might vary with size. Basically if the spectral shape changes with size then it would not be possible to get the spectra to overlie each other under any particular frequency scaling or choice of $\varepsilon$ as described in equation 5 . This would be an observational indicator of non-self-similarity.

\section{ENERGY AND MOMENT OBSERVATIONS}

\subsection{Published Studies}

As indicated in Table 1 there have been many studies that have looked into and reached conclusions about apparent stress scaling. It is beyond the scope of this paper to review them all here. Instead we want to briefly review some of the most widely used techniques as illustrated by selected published studies.

4.1.1 Borehole and Mine Studies. As indicated in Figure 6 the main challenge to investigating scaling effects in small to moderate events is the ability to see true source amplitude at high frequencies $(>10 \mathrm{~Hz})$. The biggest impediment to observing high frequencies is the strong attenuation and site effects caused by weathering and other high attenuation and scattering effects near the surface of the earth. To minimize these effects investigators have used borehole recordings of very close events to observe relatively unattenuated signals up to hundreds of $\mathrm{Hz}$ or more. The pioneering study of Abercrombie (1995) is perhaps the best known of these. There are a number of others (e.g. Prejean and Ellsworth, 2001; Ide et al., 2003). Recording in mines can similarly minimize such effects (e.g. Richardson and Jordan, (2002); Oye et al. 2005; Yamada et al., 2005). However as shown by the quite different conclusions regarding scaling reached by Stork and Ito, (2004) compared to Imanshi et al. (2004), both using data from an $800 \mathrm{~m}$ deep borehole in Japan, there remain propagation effects even in subsurface data that can preclude definitive answers.

4.2.2 Empirical Green Functions. This is perhaps the most attractive method for removing path and site effects. Find a co-located pair of earthquakes with significantly different magnitudes. Then the smaller one, due to its much shorter duration and much high corner frequency can be considered a delta function with respect to the larger one and used 
to correct back to a source time function or spectra. A nice example of an empirical Green function (EGF) time domain study, among many that are available is Mori et al (2003) which looked at apparent stress scaling in the Northridge earthquake sequence. There are many examples of frequency domain studies where spectral ratios between large and small events are used (e.g. Izutani and Kanamori, 1991; Matsuzawa et al. 2004; Izutani, 2005). Problems include lack of EGF's for some events of interest and issues of how small changes in depth, mechanism and location affect the results. Also real events are not perfect Green functions and this can also have an effect on the spectral estimate (e.g. Prieto et al., this volume).

4.2.3 Time or Frequency Domain Integration. This is the oldest and most straightforward of techniques: correct the observed data for path effects and integrate to get radiated energy. Teleseismic waveforms have been routinely used to estimate energy for many years (e.g. Boatwright and Choy, 1986; Choy and Boatwright, 1995). As high dynamic range digital data became available in the 1990's local and regional studies covering a wider range in magnitude became possible allowing studies to look at apparent stress scaling isuees. The pioneering study of Kanamori (1993), who integrated local to regional velocity squared time records corrected for path effects, is perhaps the best known of these. As many other investigators began also using regional records to determine radiated energy a discrepancy was found between the regional estimates and the teleseismic ones (e.g. Singh and Ordaz, 1994; Mayeda and Walter, 1996). Although this issue has been examined (e.g. PerezCampos, 2003) it is not yet been completely resolved.

\subsection{New Observations of the 1999 Hector Mine Sequence}

Many prior studies of earthquake scaling are affected by multiple varying parameters such as station site effects, path effects, radiation pattern corrections, etc., which can increase scatter and uncertainty in their determinations of scaled energy. Even when great care is taken to account for these effects different researchers end up with different results. The1999 Hector Mine earthquake in southern California is a good example. Boatwright et al., (2002) estimate $E_{R}$ of about $3.3 \times 10^{15} \mathrm{~J}$, whereas Venkataraman and Kanamori (2004) revised their initial estimate (Venkataraman et al., 2002) for this event downward to $1.0 \times 10^{15} \mathrm{~J}$ after correcting for finite fault effects. Thus there are baseline differences between researchers working on the same 
event, since there is no known energy reference event to calibrate to. All of these sources of uncertainty feed into compilation studies of scaled energy such as Ide and Beroza (2001). The resulting large scatter makes it impossible to determine apparent stress scaling or lack thereof.

One way to minimize site and path tradeoffs with source estimate is to examine the scaling within an earthquake sequence recorded at a constant set of stations. For a small source region relative to the station distance, the path and site effects are basically the same for each event. We can then examine the slope of the radiated energy versus moment for the sequence. This minimizes baseline issues, as we expect any such systematic effects apply to all the events of a sequence with less effect on the measured slope. In this paper we make use of the $M_{w}=7.1$ mainshock and the rich aftershock sequence of events greater $M_{w}=3.75$ to look at earthquake scaling.

4.2.1 Direct Wave Energy Estimation. Here we illustrate how the direct regional phases such as $L g$ can be used to determine radiated energy for events in a large earthquake sequence. The 1999 Hector Mine earthquake sequence in southern California was recorded on scale (without clipping) at a number of digital broadband stations in the western U.S including CMB, ELK and TUC (see figure 8). In Figure 9 we show regional $\mathrm{Lg}$ spectra recorded at station ELK for the $M_{w}=7.1$ mainshock and $M_{w}=5.3$ and $M_{w}=4.1$ aftershocks. These are 3 component spectra, averaged over the vertical, east and west components and they are truncated for frequencies where the signal-to-noise ratio drops below a value of two. We note the common path and station indicate that any spectral differences, including radiated energy, observed between the events can be ascribed to source effects. We added a closer station, ISA, to enhance the ability to see high frequencies and to have a check against distance dependent effects. While the mainshock Lg is clipped at ISA, the aftershocks are very well recorded.

To determine the seismic energy from the $\mathrm{Lg}$ spectra we need to determine an apparent attenuation common for all events recorded at that station to correct for the path and site effects. We use a Street et al. (1975) style geometrical spreading $G(R)$ : 


$$
G(R)=\left(\begin{array}{lll}
\frac{1}{R} & \text { where } & R<R_{o} \\
\frac{1}{R_{o}}\left(\frac{R_{o}}{R}\right)^{\eta} & \text { where } & R \geq R_{o}
\end{array}\right)
$$

and a power law $\mathrm{Q}$ function of frequency:

$$
Q(f)=Q_{o} f^{\gamma}
$$

assuming that this approach is adequate to fit the combined path and site apparent attenuation. Here $\mathrm{R}$ is the sourcestation distance and $R_{o}$ is a critical distance, which we take to be $100 \mathrm{~km}$ and $\eta$ is the spreading exponent, which we take to be 0.5 . We used a simple earthquake spectral model developed by Walter and Taylor (2001) to match the mainshock spectra in order to estimate the apparent Q for several independent apparent stress estimates. The model uses the geometrical spreading and frequency dependent Q shown above with a generalized Brune (1970) spectral shape that has been altered to allow variable apparent stress scaling. The model is called MDAC for Magnitude and Distance Amplitude Correction and was developed to remove such trends from earthquake-explosion discriminants. Here we use it to get an estimate of the power law Q parameters. It is important to note that once we have Q parameters for each station we use them to correct the observed aftershock spectra and determine aftershock radiated energy by integration of the Q corrected spectra without making any source model assumptions, MDAC or otherwise.

We used the estimates of the Hector Mine mainshock moment of $M_{w}=7.13$ from Ji et al., (2002), and energy from Boatwright (2002) of $3.3 \times 10^{15} \mathrm{~J}$ to determine an independent apparent stress of $1.66 \mathrm{MPa}$ for the mainshock. We then did a grid search over the Q parameters in equation 10 to determine the best fit for each of the stations. We show an example of some of the fits at station TUC in figure 10. The overall fit to the observed data is good across the entire observed frequency band. However as we have noted previously there are other energy estimates for the Hector Mine mainshock. We repeated the analysis using the energy estimate of $1.0 \times 10^{15} \mathrm{~J}$ from Anu Venkataraman (pers. comm.) based on a finite fault revision to the teleseismic study in Venkataraman et al., (2002). Along with eht Ji et al. (2002) moment this gives an apparent stress of $0.53 \mathrm{MPa}$. We label this attenuation set "Model A" and the one based on Boatwright(2002) "Model B". The Q parameters for each station and each model are given in Table 2. 
Stations CMB, ELK and TUC are determined from the mainshock Lg spectra. At station ISA we used several aftershocks in common with the 3 calibrated stations to determine the Q parameters.

Table 2. Apparent attenuation parameters for the Hector Mine Sequence to each station.

\begin{tabular}{|l|l|l|l|}
\hline \multicolumn{1}{|c|}{ Station } & \multicolumn{1}{c|}{ Model A } & \multicolumn{1}{c|}{ Model B } & \multicolumn{1}{c|}{ Model C } \\
\hline CMB & $405 \mathrm{f}^{0.25}$ & $260 \mathrm{f}^{0.40}$ & $175 \mathrm{f}^{0.44}$ \\
\hline ELK & $400 \mathrm{f}^{0.25}$ & $290 \mathrm{f}^{0.35}$ & $195 \mathrm{f}^{0.44}$ \\
\hline ISA & $1480 \mathrm{f}^{0.0}$ & $550 \mathrm{f}^{0.17}$ & $150 \mathrm{f}^{0.49}$ \\
\hline TUC & $405 \mathrm{f}^{0.38}$ & $260 \mathrm{f}^{0.52}$ & $190 \mathrm{f}^{0.52}$ \\
\hline
\end{tabular}

After fixing the path parameters based on the mainshock we determine the energy for each of the aftershocks by spectral integration over the frequency band where the signal to noise ratio is greater than two. On the low frequency side we determine an additional missing energy term by extrapolating from the lowest observed frequency to zero assuming a constant amplitude level. On the high frequency side we determine an additional missing energy term by extrapolating from the highest observed frequency to infinity assuming a falloff of $\mathrm{f}^{-2}$. Because, as we showed in figure, the energy is concentrated near the corner frequency, we must be careful that for the smaller events the corner frequency does not reach or exceed the highest frequency or our energy estimate will be incorrect. We also want to make sure we do not censor out earthquakes with high corner frequencies to bias our dataset. After a careful analysis we set a lower magnitude cutoff of Mw 3.75 and include all such events. We also require at least 3 of the 4 stations to have energy estimates to minimize any azimuthal or radiation pattern effects and to get a more robust energy determination. In Figure 11 we compare energy estimates for Model B at some of the different stations to show that we are not getting biased results from a given station and that there are not obvious distance or azimuthal trends in the results.

We plot the Lg-based energy estimates versus coda-based seismic moments for all the events for each of the three different attenuation models in Figure 12. The slope of the best fit line to the data for each model gives the apparent stress scaling. Constant apparent stress or self-similar scaling would give a slope of 1.0. Slopes greater than 1 indicate that apparent stress is increasing with moment in viola- 
tion of self-similarity. The Model B corrected data show non-self-similar behavior with scaled energy varying with moment as $0.20 \pm 0.06$, which means that $\varepsilon=0.75 \pm 0.28$ as defined in equation (8). The results for Model A show a similar but slightly stronger non-self-similar scaling. The attenuation parameters for Model $\mathrm{C}$ were derived by choosing a high enough apparent stress for the main shock that the resulting attenuation function would give slope that was equal to one within the uncertainty giving a self-similar model. This turned out to require assuming a fairly high apparent stress for the mainshock. As can be seen in figure 12 the apparent stress for the sequence still shows some slight scaling but it has a slope of one to within the standard deviation. If this model were correct, the apparent stresses for all events would be quite high, averaging around $100 \mathrm{MPa}$ and the apparent stress of the main shock would be very high, about $13 \mathrm{MPa}$, which is inconsistent with the independent estimates of Boatwright (2002), Vekatamaran et al. (2002) and others. In addition, the results of Model C are not consistent with our independent results using regional coda or spectral scaling as we will discuss in the next two subsections. Therefore our preferred result is the one obtained using the attenuation Model B.

4.2.2 Regional Coda Wave Energy Estimation. We have previously published (Mayeda et al. 2005) an analysis of the 1999 Hector Mine sequence using regional coda wave analysis. Here we just briefly review the coda methodology in comparison with using direct waves. The coda results provide an independent check on the direct wave results. As we will show the coda energy estimates are most consistent with the Model B direct wave results.

Regional seismic $S$-wave coda consists of the scattered wavefield following the direct $L g$ arrival. If the envelopes of the seismic trace are taken in a variety of narrow passbands, estimates of the coda spectra can be derived by fitting the envelopes. By correcting these spectra for path, site, and $S$-wave-to-coda transfer function effects, a codabased source spectra can be derived. This regional coda envelope technique for source spectra has evolved over the past decade (Mayeda, 1993; Mayeda and Walter 1996; Mayeda et al., 2003). The most recent version of the methodology has been developed to be completely empirical and independent of assumptions about scattering models. 
We have compared direct waves measures with coda waves (e.g., Mayeda, 1993; Mayeda and Walter, 1996; Mayeda et al., 2003) and find that amplitude measurements of direct waves requires significant multi-station averaging to achieve the same stability as a single coda envelope measurement. Specifically, the stability comes from the fact that: (1) the coda samples a significant part of the focal sphere, in contrast to the direct waves which sample limited take-off angles; (2) the coda envelope amplitude represents a cumulative effect of the entire rupture process, effectively averaging over the source-time function; (3) the scattered wavefield effectively averages over lateral heterogeneities; (4) the simultaneous fit to the observed envelope over a large portion of time minimizes measurement error that affects short-window length direct wave measurements. In general, the studies mentioned previously find that the source amplitude obtained from the coda envelope is a factor of 3-to-4 times more stable than those derived from direct waves. In other words, a single coda envelope measurement is equivalent to a 9-to-16-station average using direct waves.

In an early general study using the coda methodology for earthquakes scattered around the western U.S Mayeda and Walter (1996) found apparent stress scaling with moment approximately as 0.25 or as $\varepsilon=1$ in terms of equation (8). We recently applied the updated coda methodology of Mayeda et al 2003 to the Hector mine sequence at the same four stations of CMB, ELK, ISA, and TUC shown in Figure 8 . The coda based energy results are given in Figure 3 of Mayeda et al. (2005) and show apparent stress scaling as $0.176 \pm 0.05$ or $\varepsilon=0.64 \pm 0.23$ in terms of equation (8). This result is quite similar to the direct Lg results for Model B discussed in the previous section. In Figure 13 we compare the direct $\mathrm{Lg}$ and the coda based energy estimates for the Hector Mine sequence. The coda results are most consistent with the Model B results and quite different form the energy estimates derived using the direct waves from Models A and C. It appears that using Lg Q terms very slightly more attenuative than Model B we could get even better match between the direct waves and coda. We also note that the direct $\mathrm{Lg}$ results for Model B and the coda results are essentially equal in terms of their predictions of scaling when the uncertainty is taken into account. Thus using two different methods, a direct $\mathrm{Lg}$ and a coda technique, we reach similar conclusions that the scaling within the 1999 Hector Mine sequence in non-self-similar.

4.2.3 Spectral Scaling. As discussed in section 3.2 and 3.3 the shape of the inferred source spectra under a frequency transformation of the form in equation (5) can be used to assess if earthquake scaling is self-similar or not. This 
property was pointed out by Prieto et al (2004), who used it to determine that a sequence of small earthquakes (magnitude 1.8 to 3.4 ) in Southern California was self-similar. We have also applied this technique to the coda spectra derived source spectra for the Hector Mine earthquake sequence in Mayeda et al. (2005). The results are shown in figures 4 and 5 of that paper and indicate that a scaling of $f$ ${ }^{3.5}$ produces much more self-consistent results than $f^{3}$. Such a spectral scaling implies that $\varepsilon$ is about 0.5 , which is consistent with the integrated energy results for coda and direct waves taking into account the uncertainty of those measures.

In this paper we look at the spectral scaling of the $\mathrm{Lg}$ derived source spectra using the attenuation models discussed in section 4.2.1. Figure 14 shows the $\mathrm{Lg}$ spectra from the same three Hector Mine events recorded at station ELK as shown if Figure 9, except they have been corrected for geometrical spreading, path and site effects to give inferred moment rate source spectra. We can see that the two different attenuation Models $\mathrm{B}$ and $\mathrm{C}$ give slightly different shapes to the inferred source spectra. We can make use of equation (5) to see how well the spectra will match each other under frequency transformations for different values of epsilon. The integrated spectral energy results for Model B indicated that the data should be consistent with a scaling using an $\varepsilon=0.75 \pm 0.28$. The results using $\varepsilon=0.5$ are shown in Figure 14c and there is some consistency in the scaled spectra with each other. The parameters for Model $\mathrm{C}$ were constructed to give the least attenuative model that was consistent with an $\varepsilon=0$ model, within the uncertainty. In Figure 14d the Model $\mathrm{C}$ inferred source spectra are frequency scaled using $\varepsilon=0$ and the results show less consistency than the Model $\mathrm{B}$ case. This result implies that if the Model $\mathrm{C}$ attenuation parameters were correct, the spectral shapes themselves do not appear to be self-similar even though the integrated energy results can be interpreted this way. This is possible if the source spectral shapes change with size as discussed in section 3.3.2 while having constant scaled energy.

\subsection{DISCUSSION}

Considering all the studies of apparent stress scaling to date, and looking for interpretations that might be consistent with most of them we can construct a number of hypothetical models as shown in Figure 15. The first possibility is that apparent stress is constant when a large enough set 
of earthquakes is looked at but overall the variance is very large as shown in Figure 15a. If this model were correct it raises the question of what is the source of the large variability. Is it due to large differences in initial and boundary conditions for different faults in different regions or is the rupture process itself somewhat chaotic with small changes in initial conditions able to result in big changes in apparent stress? This is an important area of investigation even if earthquake apparent stress does scale with size.

A second possibility is that apparent stress scales with size, with a somewhat smaller variance is shown in Figure 15b. Here we show a scaling of $\varepsilon=0.5$ or $\tilde{e}=M_{o}^{0.14}$ as we found for the 1999 Hector Mine sequence and has been observed for in other earthquake sequences (e.g. Mayeda et al., 2005). If this model is correct it raises the question of what physics is causing radiated energy efficiency to increase with size. A third possibility is that there is an apparent stress level difference between small and large events with a transition zone in between as shown in Figure 15c. In this model studies of small earthquakes (e.g. magnitude $<$ 3.5) or large earthquakes (e.g. magnitude $>6$ ) would observe constant apparent stress. For mid-range magnitudes the earthquakes undergo a transition from a lower to a higher apparent stress level. This kind of transitional model raises questions of why such a transition should occur. Possibilities include threshold type behavior when rupture exceeds a critical size and the physics changes at the small magnitude end. At the large magnitude end one might invoke boundary condition changes such as a change from equdimensional growth to uni-dimensional growth as rupture approaches the depth of the seismogenic zone.

A fourth possibility is that scaling is a common feature of sequences that occur on particular faults or within small regions. In this hybrid model different faults or regions would have scaling behavior with maximum magnitudes and apparent stress levels determined by the local conditions as shown in Figure 15d. If a large amount of data was looked at together this model might look a lot like the constant apparent stress model in Figure 15a, but with the important difference that within a sequence there would be predictable scaling which has utility for hazard prediction.

All of the models in Figure 15 indicate that small events could have quite different apparent stress values than large events, either due to variance or scaling effects. Therefore this effect needs to be taken into account when small events are used to predict the ground motions of large events for hazard studies. Clearly approaches that can reduce the variance of individual estimate of radiated energy and inferred 
source spectra are quite important in this regard.

To differentiate between the different model possibilities multiple techniques applied systematically to many earthquake datasets will be needed. In this regard well studied earthquakes such as the 1999 Hector Mine sequence can serve as benchmarks to validate techniques against. This would help eliminate the possibility that some of the large differences observed between studies are due to differences within the techniques themselves .

Acknowledgments. We thank the Chapman conference organizers for arranging such a stimulating workshop and inviting us to prepare and present this paper. Some of this work was supported by LLNL lab-wide LDRD funding. This work was performed under the auspices of the U.S. Department of Energy by the University of California, Lawrence Livermore National Laboratory under contract No. W-7405-Eng-48.

\section{REFERENCES}

Abercrombie, The start of something big?, Nature 438, 171-172, 2005.

Abercrombie, R. E. (1995). Earthquake source scaling relationships from -1 to $5 M_{L}$ using seismograms recorded at $2.5 \mathrm{~km}$ depth, J. Geophys. Res., 100, 24,015-24,036.

Beeler, N. M., T.-F. Wong and S. H. Hickman (2003). On the expected relationships among apparent stress, static stress drop, effective shear fracture energy and efficiency, Bull. Seism. Soc. Am, 93, 1381-1389.

Boatwright, J., G. L. Choy and L. C. Seekins, (2002). Regional estimates of radiated energy, Bull. Seism. Soc. Am, 92, 12411255.

Boatwright, J. and G. L. Choy (1986). Teleseismic estimates of the energy radiated by shallow earthquakes, J. Geophys. Res., 91, 2095-2212.

Boatwright, J. and J. B. Fletcher (1984). The partition of radiated energy between $\mathrm{P}$ and S waves, Bull. Seism. Soc. Am., 74, 361376.

Brodsky, E. E. and H. Kanamori (2001). Elastohydrodynamic lubrication of faults, J. Geophys. Res. 106, 16,357-16,374.

Brune, J., S. Brown and P. Johnson (1993), Rupture mechanisms and interface separation in foam rubber models of earthquakes: a possible solution to the heat flow paradox and the paradox of large overthrusts, Tectonophysics, 218, 59-67.

Choy, G. L. and J. L. Boatwright (1995). Global patterns of radiated seismic energy and apparent stress, J. Geophys. Res. 100, 18,205-18,228.

Dreger, D. (1994), Empirical Green's Function Study of the January 17, 1994 Northridge, California Earthquake, Geophys. Res. Lett., 21, 2633-2636.

Dreger, D., J. Ritsema, and M. Pasyanos (1995), Broadband Analysis of the 21 September, 1993, Klamath Falls Earthquake 
Sequence, Geophys. Res. Lett., 22, 997-1000.

Dreger, D. (1997), The Large Aftershocks of the Northridge Earthquake and their Relationship to Mainshock Slip and Fault Zone Complexity, Bull. Seism. Soc. Am., 87, 1259-1266.

Eken, T., K. Mayeda, A. Hofstetter, R. Gök, G. Örgülü, N. Turkelli (2004), An application of the coda

methodology for moment-rate spectra using broadband stations in Turkey, Geophys. Res. Lett, 31, Art. No. L11609.

Favereau, P. and R. J. Archuleta (2003). Direct seismic energy modeling and application to the 1979 Imperial Valley earthquake, Geophys. Res. Lett., 30, Article No. 1198.

Heaton, T. (1990). Evidence for and implications of self-healing pulses of slip in earthquake rupture, Phys. Earth Planet. Int., 64, 1-20.

Hough, S. E., D. S. Dreger (1994), Source Parameters of the 4/22/92 M6.1 Joshua Tree, California Earthquake and its Aftershocks: Empirical Green's Function Analysis of GEOS and TERRAscope Data, Bull. Seism. Soc. Am., 85, 1576-1590.

Ide, S. and G. C. Beroza (2001). Does apparent stress vary with earthquake size?, Geophys. Res. Lett. 28, 3349-3352.

Ide, S. G. C. Beroza, S. G. Prejean and W. L. Ellsworth (2003). Earthquake scaling down to M1 observed at $2 \mathrm{~km}$ depth in the Long Valley caldera, California, J. Geophys Res. 108, Article no. 2271.

Izutani, Y. and H. Kanamori (2001). Scale-dependence of seismic energy-to-moment ratio for strike-slip earthquakes in Japan, Geophys. Res. Lett. 28, 4007-4010.

Ji, C., D. J. Wald and D. V. Helmberger (2002). Source description of the Hector Mine, California earthquake, Part II: complexity of slip history, Bull. Seism. Soc. Am, 92, 1208-1226.

Jin, A. and E. Fukuyama (2005). Seismic energy for earthquakes in southwest Japan, Bull. Seism. Soc. Am., 95, 1314-1333.

Kanamori, H. K. and D. L. Anderson (1975). Theoretical basis of some empirical relations in seismology, Bull. Seism. Soc. Am., 65, 1073-1095.

Kanamori, H., J. Mori, E. Hauksson, T. H. Heaton, L. K. Hutton, and L. M. Jones (1993). Determination of earthquake energy release and $M_{L}$ using TERRAscope, Bull. Seism. Soc. Am. 83, 330-346.

Kanamori, H. and T. H. Heaton (2000). Microscopic and macroscopic physics of earthquakes, in Geocomplexity and the Physics of Earthquakes, J. Rundle, D. L. Turcotte and W. Kein (Editors), American Geophysical Union Monograph 120, 147-155.

Kanamori H. and L. Rivera (2004). Static and dynamic scaling relations for earthquake and their implications for rupture speed and stress drop, Bull. Seism. Soc. Am., 94, 314-319.

Kaverina, A., D. Dreger, and M. Antolik (1998). Source process of the 21 April, 1997, Santa Cruz Island Earthquake (Mw 7.8), Geophys. Res. Lett., 25, 4027-4030.

Jeffreys, H. (1942). On the mechanics of faulting, Geol. Mag., 79, 291.

Malagnini, L., K. Mayeda, A. Akinci, P.L. Bragato (2004). Estimating absolute site effects, Bull. Seismol. Soc. Am., 94, 13431352.

Mayeda, K. (1993), $\mathrm{m}_{\mathrm{b}}\left(\mathrm{L}_{\mathrm{g}}\right.$ coda): A stable single station estimator of magnitude. Bull. Seismol. Soc. Am. 83, 851-861.

Mayeda, K. and W. R. Walter (1996). Moment, energy, stress drop and source spectra of western United States earthquakes 
from regional coda envelopes, J. Geophys. Res., 101, 11,19511,208 .

Mayeda, K., A. Hofstetter, J. L. O'Boyle and W. R. Walter (2003). Stable and transportable regional coda magnitudes based on coda-derived moment rate spectra, Bull. Seism. Soc. Am. 93, 224-239.

Morasca, P., K. Mayeda, L. Malagnini, and W. R. Walter (2004), Coda-derived source spectra, moment magnitudes, and energymoment scaling in the western Alps, Geophys. J. Int., (in press).

Mori, J., S. Hartzell (1990). Source inversion of the 1988 Upland earthquake: determination of a fault plane for a small event, Bull. Seism. Soc. Am., 80, 507-518.

Melosh, J. (1979). Acoustic fluidization: a new geologic process?, J. Geophs. Res., 84, 7512-7520.

McGarr, A. (1999). On relating apparent stress to the stress causing earthquake fault slip, J. Geophys. Res. 104, 3003-3001.

Olsen, E. L and R. M. Allen, The deterministic nature of earthquake rupture, Nature, 438, 212-215, 2005.

Oye, V. H. Bungum, M. Roth (2005). Source parameters and scaling relations for mining-related seismicity withn the Pyhasalmi ore mine Finland, Bull. Seism. Soc. Am. 95, 1011-1026.

Pasyanos, M.E., D.S. Dreger, and B. Romanowicz, Toward RealTime Estimation of Regional Moment Tensors, Bull. Seism. Soc. Amer., 86, 1255-1269, 1996.

Perez-Campos, X., S. K. Singh, and G. C. Beroza, (2003). Reconciling teleseismic and regional estimates of seismic energy, Bull. Seism. Soc. Am., 93, 2123-2130.

Prejean, S. and W. L. Ellsworth (2001). Observations of earthquake source parameters at $2 \mathrm{~km}$ depth in the Long Valley caldera, eastern California, Bull. Seism. Soc. Am., 91 165-177.

Prieto, G. A., P. M. Shearer, F. L. Vernon, and D. Kilb (2004), Earthquake source scaling and self-similarity estimation from stacking $\mathrm{P}$ and S spectra, J. Geophys. Res., 109, Art. No. B08310.

Richardson, E. and T. H. Jordan (2002). Seismicity in deep gold mines of south Africa: implications for tectonic earthquakes, Bull. Seism. Soc. Am. 92, 1766-1782.

Ritsema, J. and T. Lay, Long period regional wave moment tensor inversion for earthquakes in the western United states, J. Geophys. Res. 100, 9853-9864, 1995.

Romanowicz, B., and L. Ruff (2002). On the moment-length scaling of large strike-slip earthquakes and the strength of faults, Geophys. Res. Lett., 29, 1604-1608.

Roumelioti, Z., D. Dreger, A. Kiratzi, and N. Theodoulidis (2003), Slip distribution of the 7 September 1999 Athens earthquake inferred from an empirical Green's function study, Bull. Seism. Soc. Am., 93, 775-782.

Sibson, R. (1973). Interactions between temperature and porefluid pressure during earthquake faulting and a mechanism for partial or total stress relief, Nature Phys. Sci., 243, 66-68.

Singh, S. K. and M. Ordaz (1994). Seismic energy release in Mexican subduction zone earthquakes, Bull. Seism. Soc. Am., 84 1533-1550.

Street, R. L., R. Herrmann, and O. Nuttli (1975). Spectral characteristics of the $\mathrm{Lg}$ wave generated by central United States earthquakes, Geophys. J. R. Astron. Soc. 41, 51-63.

Tajima, F., K. M. Mayeda, D. S. Dreger, and G. Wurman (2002). 
Calibration for coda derived moment magnitude using Berkeley complete waveform moment-tensor solutions, Eos Trans. $A G U$, 83(47), Fall Meet. Suppl., Abstract S72B-1150.

Takahashi, T., H. Sato, M. Ohtake and K. Obara (2005). Scale dependence of apparent stress for earthquakes along the subducting pacific plate in northeastern Honshu, Japan, Bull. Seism. Soc. Am., 95, 1334-1345.

Venkatamaran, A. and H. Kanamori (2004). Effect of directivity estimates on radiated seismic energy, J. Geophys. Res. 109, doi:10.1029/2003JB002548.

Venkatamaran, A., L. Rivera, and H. Kanamori, (2002). Radiated energy from the 16 October 1999 Hector Mine earthquake: regional and teleseismic estimates, Bull. Seism. Soc. Am, 92, 1256-1265.

Walter, W. R. and S. R. Taylor (2001). A revised magnitude and distance amplitude correction (MDAC2) procedure for regional seismic discriminants: theory and testing at NTS, Lawrence Livermore National Laboratory Report, UCRL-ID-146882, http://www.1lnl.gov/tid/lof/documents/pdf/240563.pdf

Wyss, M., Stress estimates of South American shallow and deep earthquakes, J. Geophys. Res., 75, 1529-1544, 1970

Yamada, T., J. Mori, S. Ide, H. Kawakata, Y. Iio and H. Ogasawara (2005). J. Geophys. Res. 110, doi:10.1029/2004JB003221.

Mailing address for each of the authors, with paragraph indent, alphabetical order. 


\section{Time Domain}

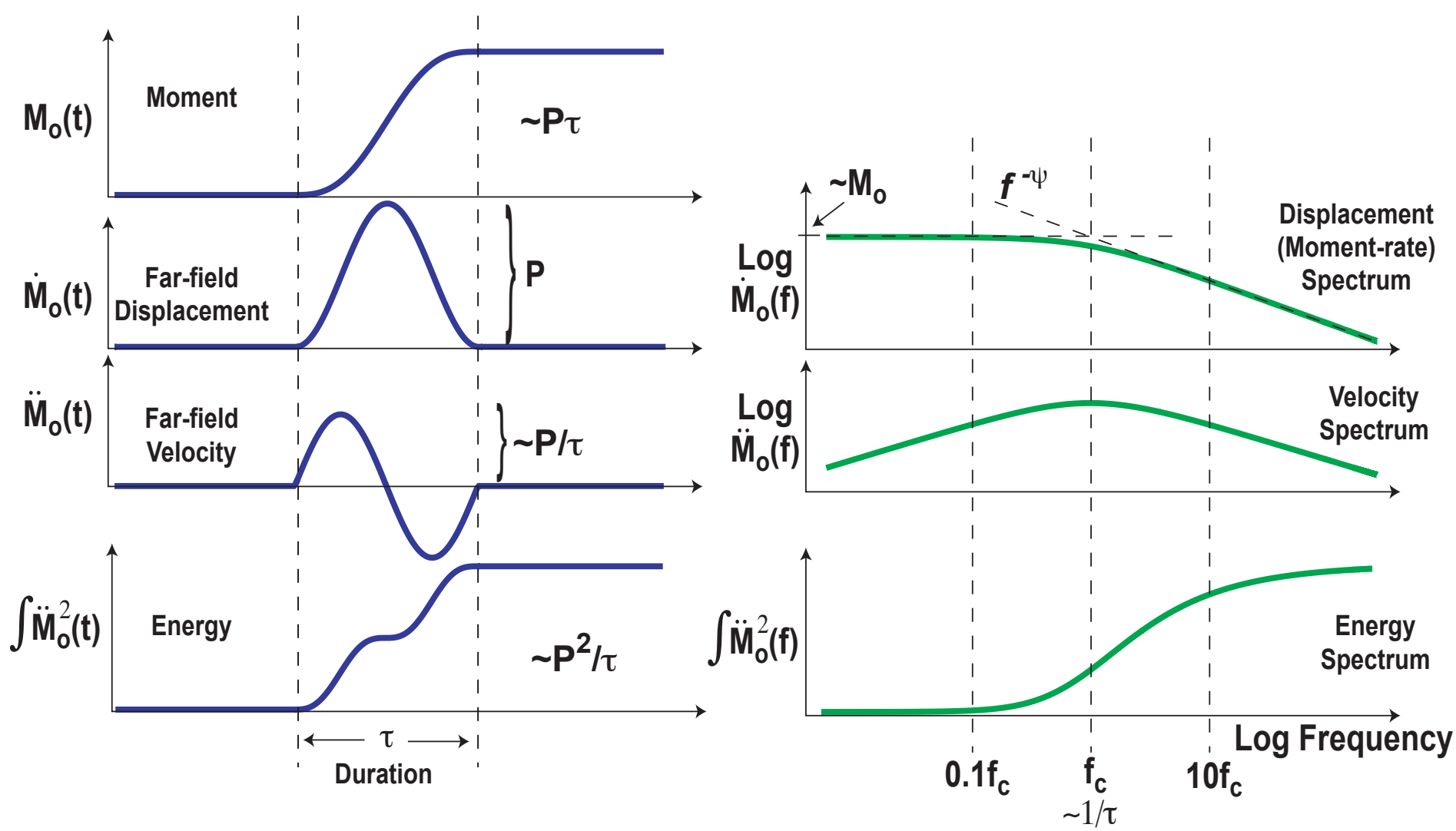

Figure 1. Simple earthquake models in the time and frequency domain. The shape of the moment time function shown here is arbitrary and the scaling relation apply for any chosen shape that has a net moment occurring over a limited duration. See text for discussion. 


\section{Quake}

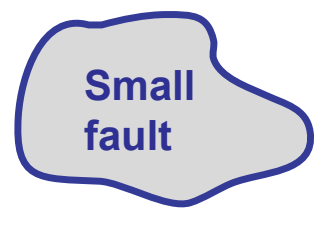

$|\leftarrow L \rightarrow|$
Scaled Quake

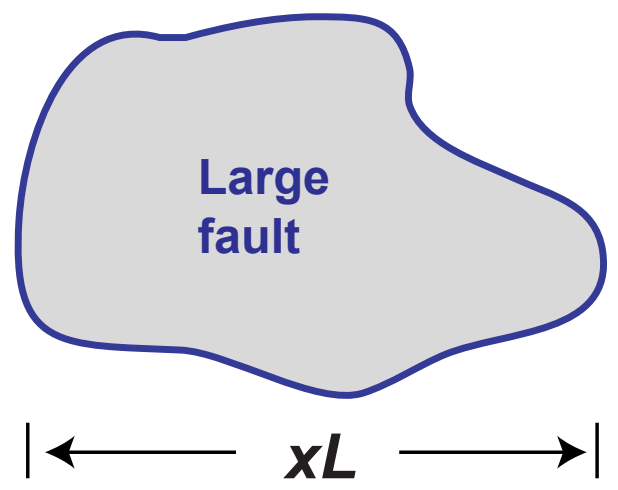

Area $=x^{2} A$

Slip $=x D$

$M_{0}=x^{3} M_{0}$

Duration $=x \square$

Energy $=x^{3} E$

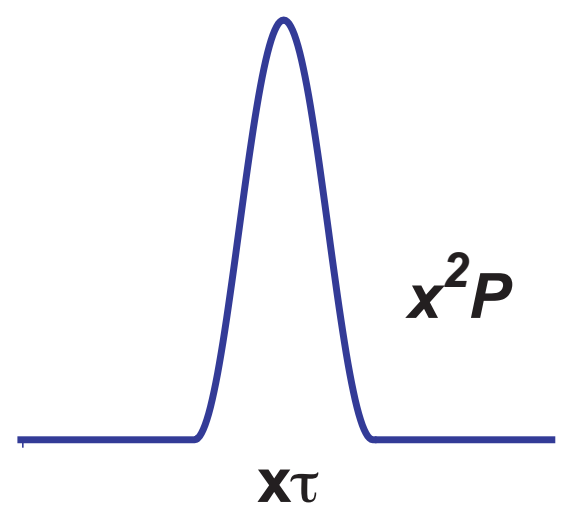

Figure 2. Self-similar scaling of an earthquake fault and associated parameters. Left hand side is the original fault and the right hand side depicts the results of scaling the fault up (or down) in size by a factor of $x$. Top depicts the arbitrary fault shape with characteristic dimension $L$. The middle show several earthquake properties dependence on $L$ and therefore how they scale with $x$. The bottom shows the scaling of the moment rate function and thus the far-field displacements. 


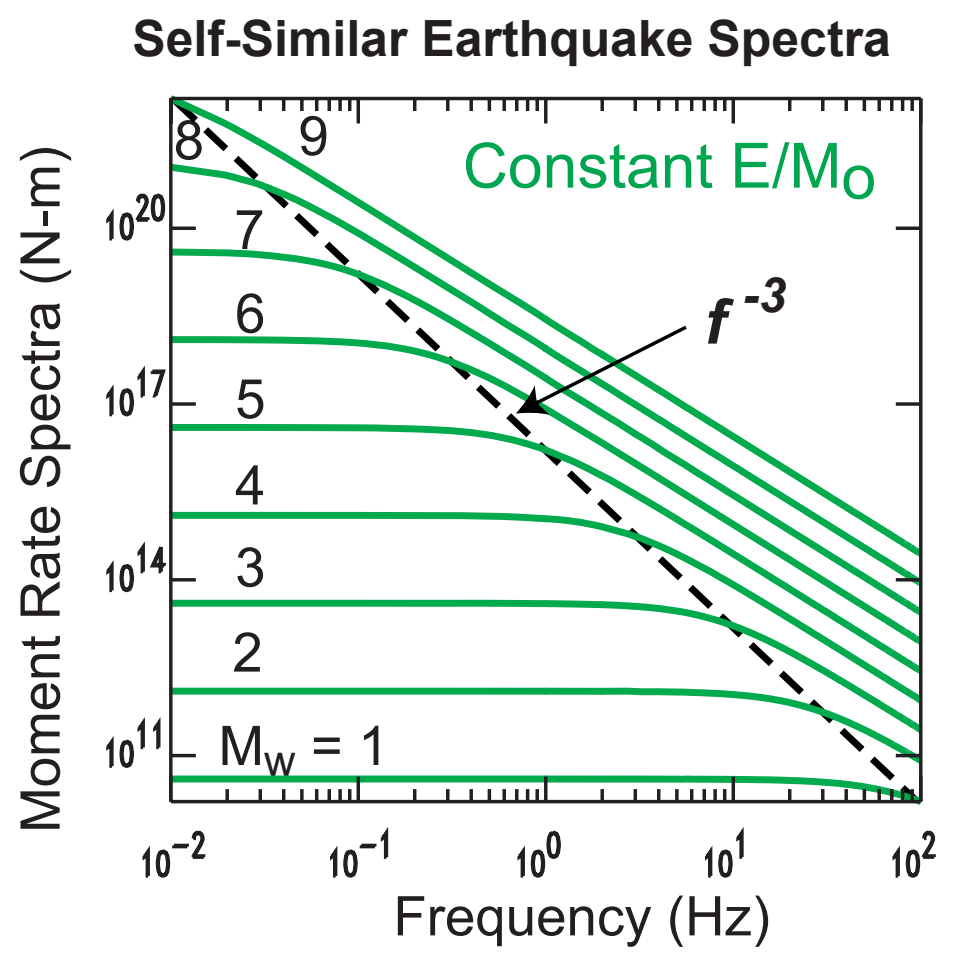

Figure 3. Self-similar model in the frequency domain for moment magnitude Mw 1 to 9. The high frequency decay rate is arbitrary. The corner frequencies lie along a line with slope $f^{3}$. As discussed in the text, the spectra can be scaled or slid along a line with this slope and shown to overlie each other. Well known examples of self-similar models of these types are given by Aki (1967) and Brune (1970). 


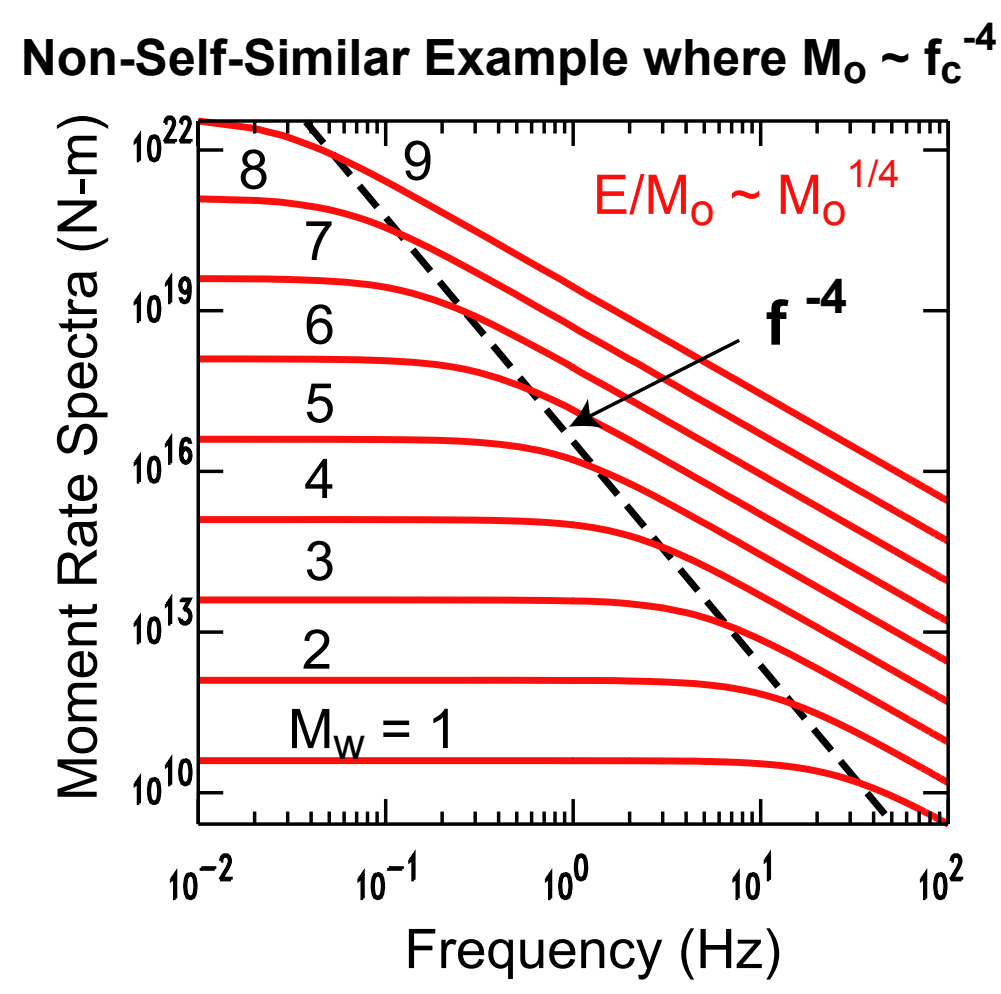

Figure 4. An example of a non-self similar model in which the shape of the spectra do not change but instead follow a different frequency scaling. In this example the corner frequencies follow a line with slope $f^{-4}$. In this case the spectra are invariant under transformations along lines with this slope. In the notation of Kanamori and Rivera (2004) this is the case of $e=1$ and leads to scaled energy varying with moment to the 0.25 power. 


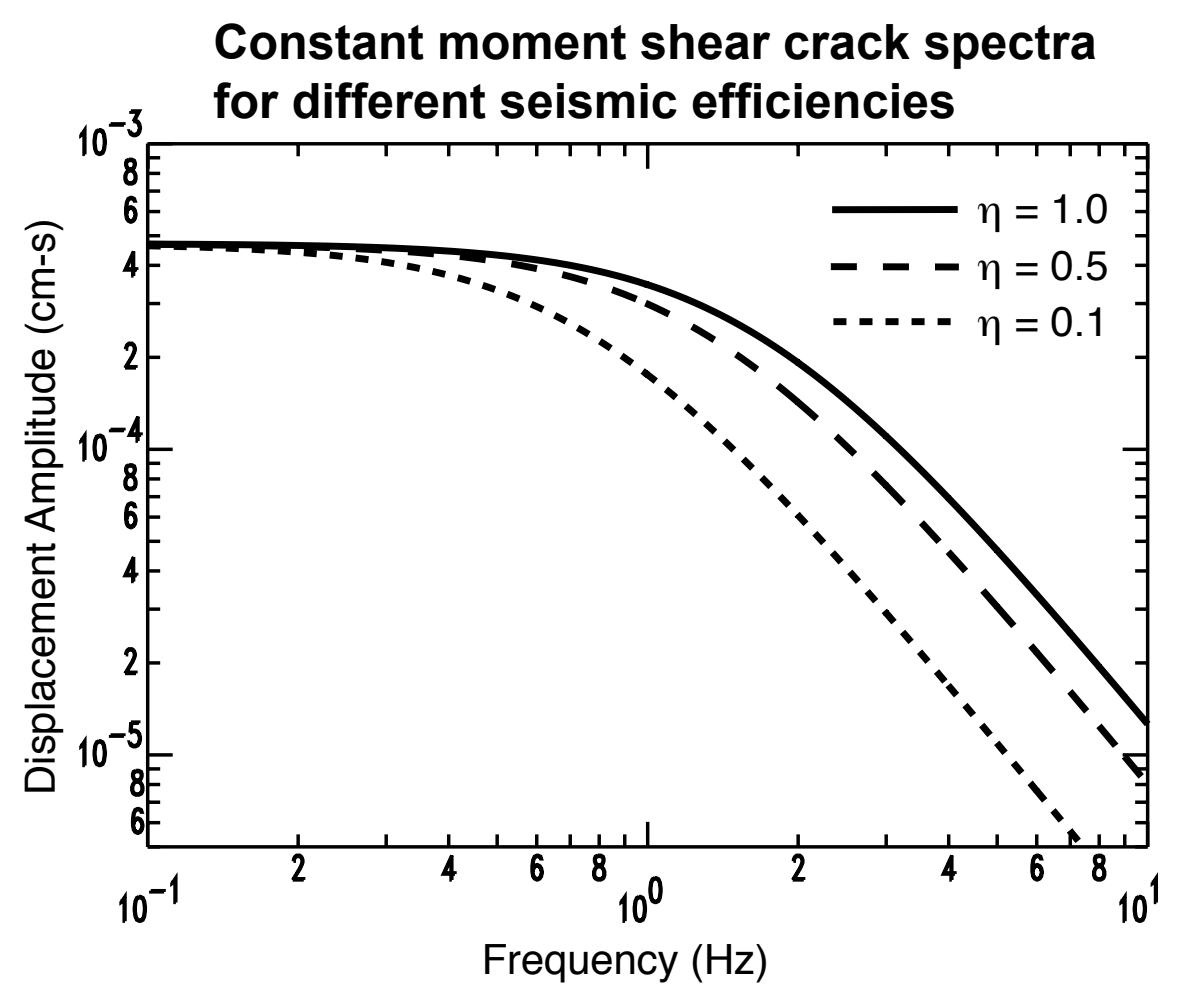

Figure 5. An example of the effect of seismic efficiency ( $\square$ ) on a constant moment circular shear fault. Note as the efficiency decreases the corner frequency decreases. If efficiency changes systematically with moment then it could cause corner frequency scaling to deviate from the self-similar expectation of cube root scaling. This particular circular shear crack model was taken from Walter and Brune (1993). 


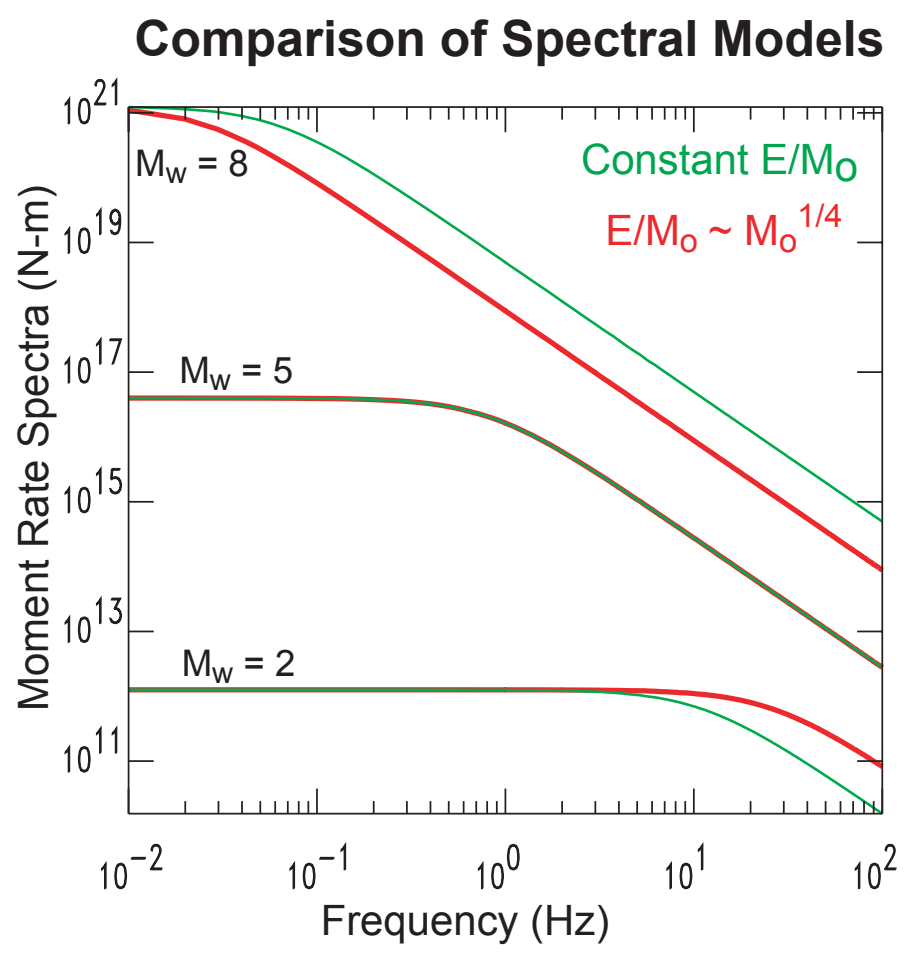

Figure 6 A comparison of the self similar model shown in Figure 3 with the non-self-similar model shown in Figure 4 at three different moment magnitudes. Here we have arbitrarily made them agree at Mw 5 . At small magnitudes the differences between the models occur at high frequencies $>10 \mathrm{~Hz}$. However the source amplitudes of such frequencies are subject to large site and path corrections. At large magnitude there is an overall change in level of amplitude over a large frequency range but such large events are relatively infrequent. 
Non-Self-Similar Example with Intermediate Falloff

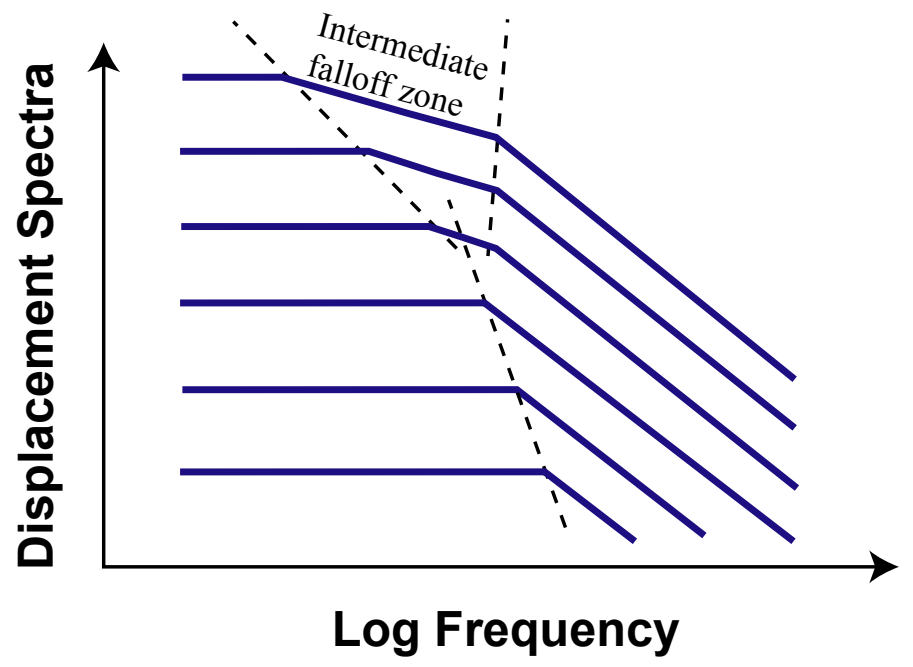

Figure 7. An example of a non-self similar model in which the shape of the spectra changes with size. In this example the larger events have an intermediate slope, which increases in bandwidth as earthquake size increases. Such an effect could occur due to change from equidimensional to unidimensional growth, or due to partial stress drop above a magnitude threshold. 
Magnitude 4+ Earthquakes from 1998-2002

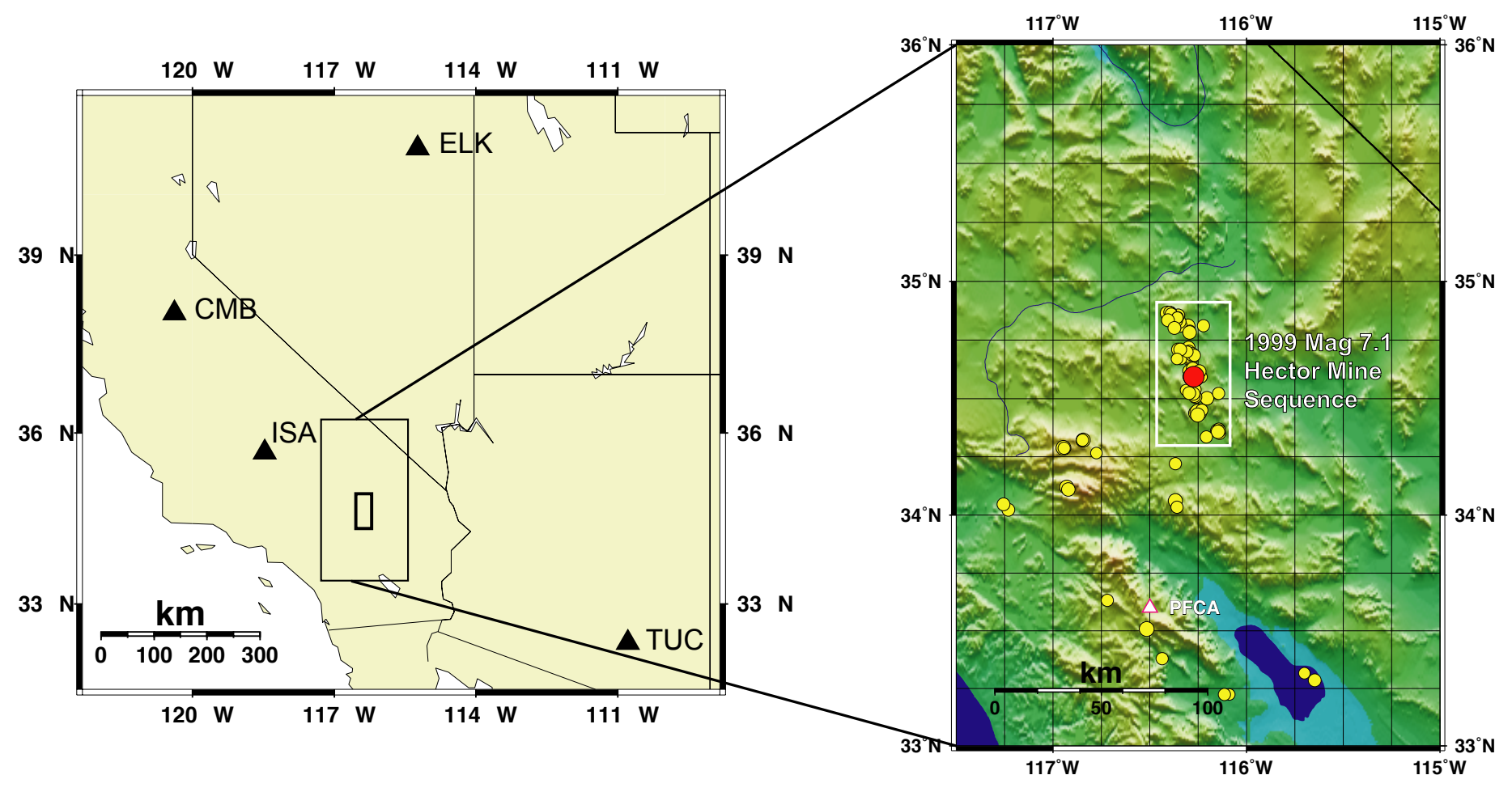

Figure 8. A map showing the location of the seismic stations and the 1999 Hector Mine earthquake sequence. The inset shows the location of all magnitude 4 earthquakes from 1998-2002. The study was limited to events within the white box. 


\section{Three-component Lg spectra at ELK}

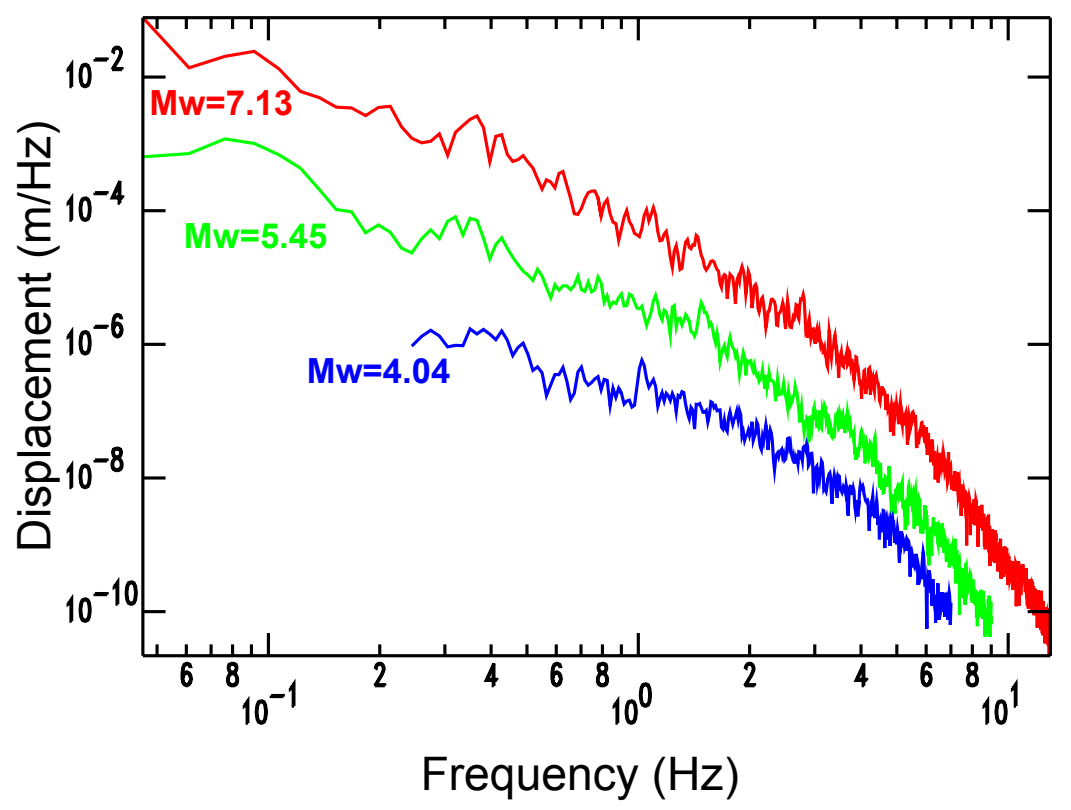

Figure 9. Three component averaged Lg spectra of the Hector Mine main shock and two aftershocks at station ELK. 


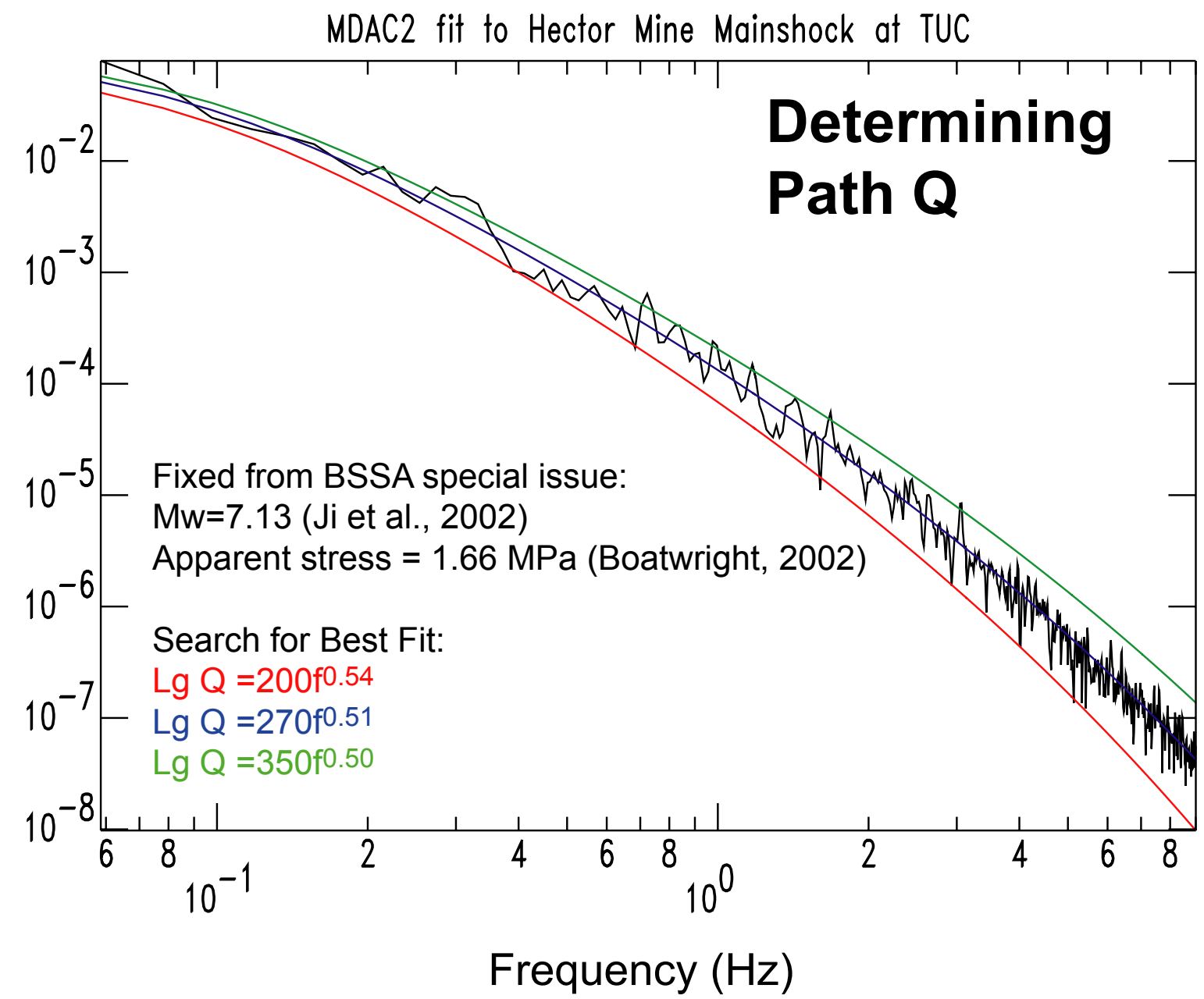

Figure 10. An example of the grid search fit for total apparent attenuation at station TUC. The best fit of $Q(f)=270 \mathrm{f} 0.51$ is shown along with a lower and higher value. Note the best fit does a good job of matching the spectrum at all frequencies. This attenuation term is then applied to all events recorded at TUC. 

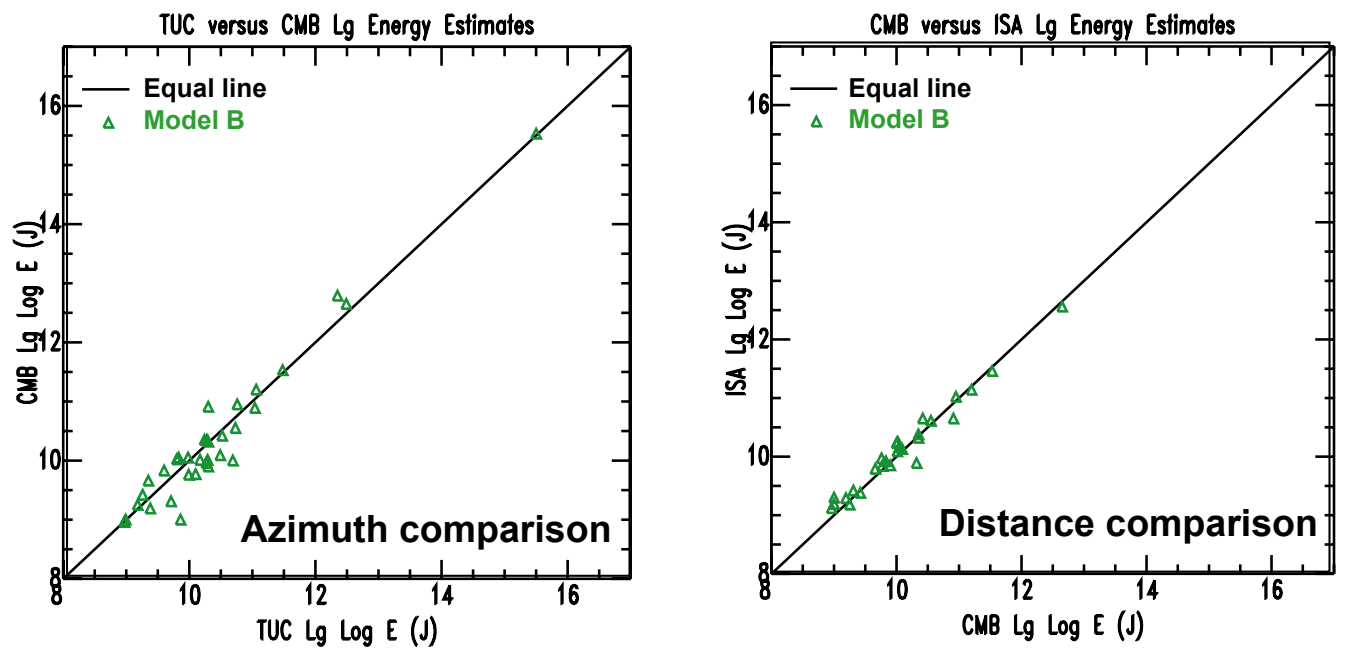

Figure 11. We look at azimuthal and distance trends in the energy estimate by comparing the results at different stations. To look at azimuthal and radiation pattern effects we compare energy estimates at TUC and CMB. While we see some scatter there does not appear to be a bias. To make sure our path corrections are reasonable we compare energy estimates at ISA and CMB, which have a similar azimuth but significantly different distances to the Hector Mine events. Again we do not see significant bias between station estimates. 


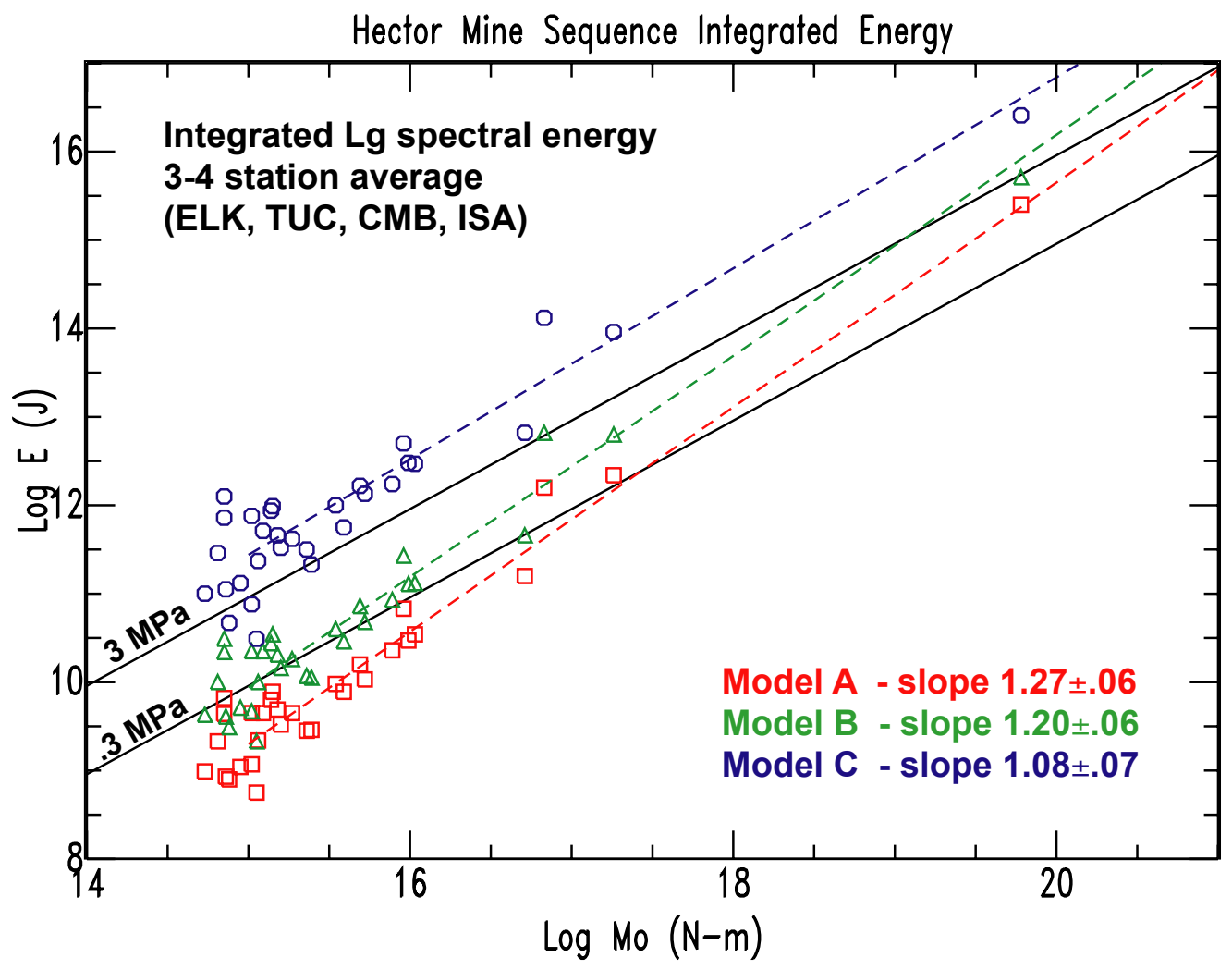

Figure 12. Here we plot the energy estimates versus the moment and measure the slope to determine the apparent stress scaling for the three different attenuation models discussed in the text. Lines of constant apparent stress are shown for reference. Our preferred results are for attenuation Model B and show apparent stress scaling with moment inconsistent with a self similar model. 


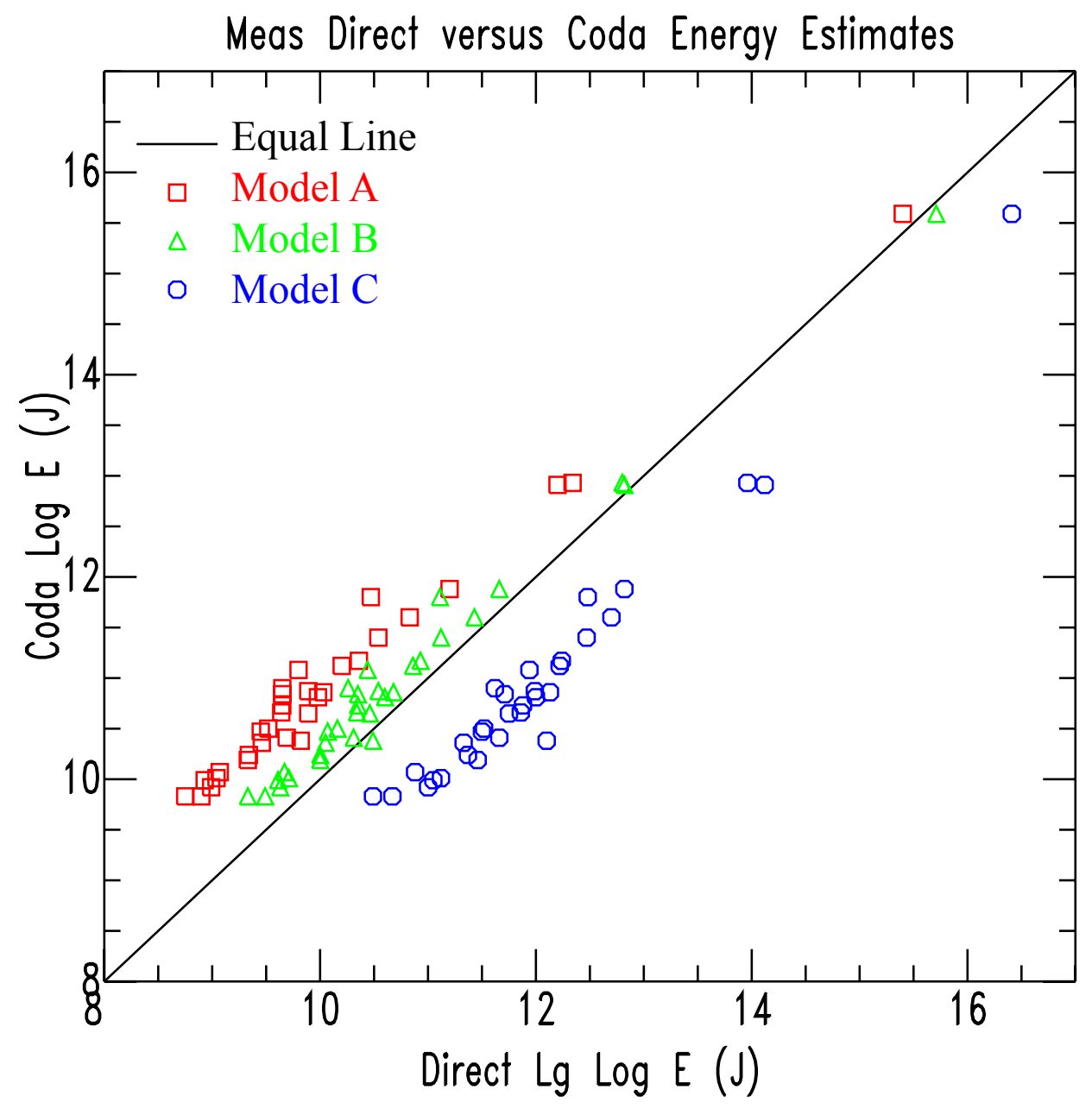

Figure 13. A comparison of the coda versus direct Lg based energy estimates for the Hector Mine sequence events. The coda results and the Model B direct Lg results are the most compatible. Each is consistent with apparent stress increaseing with moment, a non-self-similar scaling. 

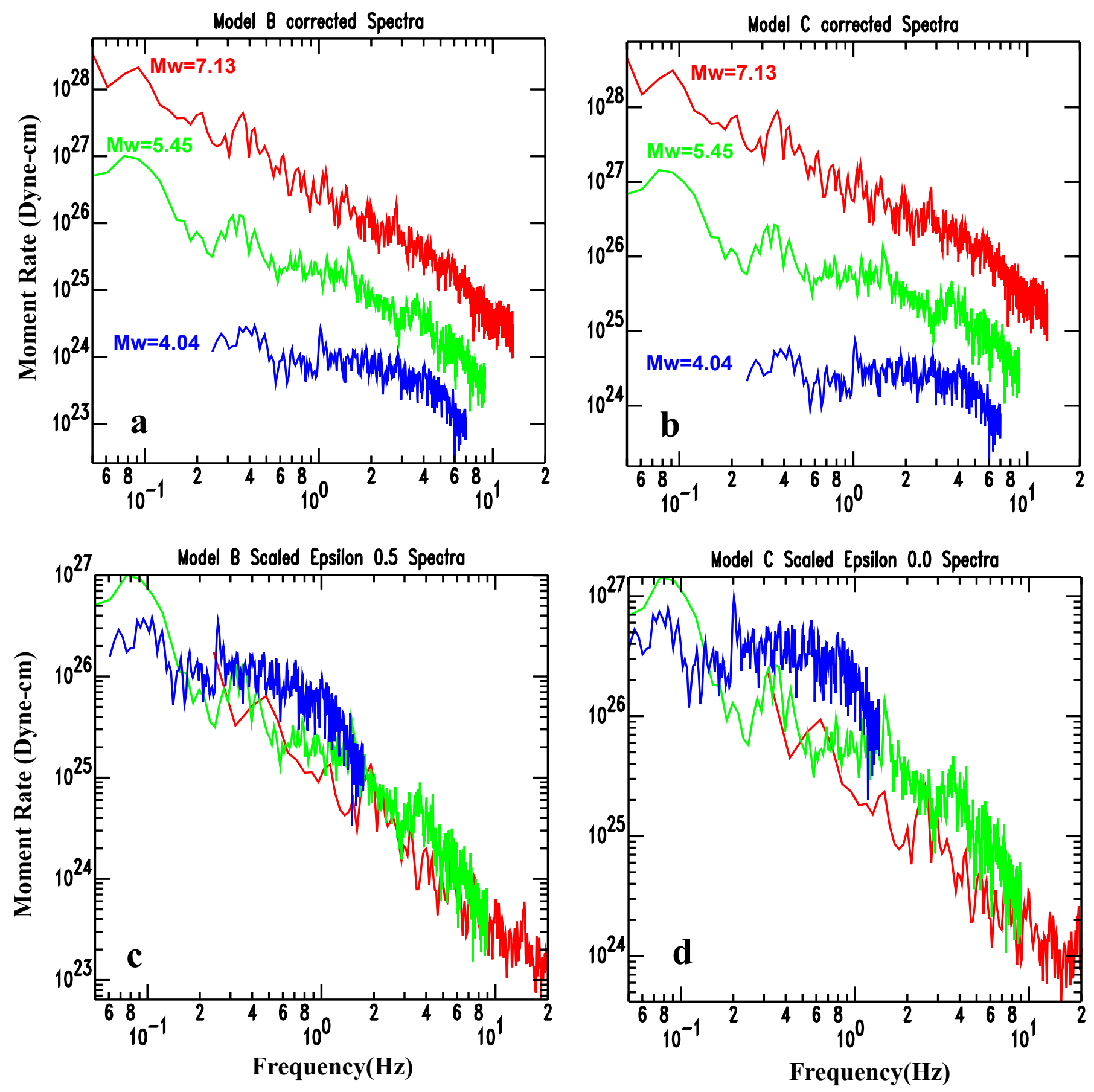

Figure 14. Same Hector Mine events as in figure 9 but corrected for geometrical spreading and attenuation to be inferred source spectra. (a) Events corrected using Model B parameters. (b) events corrected using Model $\mathrm{C}$ parameters. We scale the larger and samller events to the have $\mathrm{Mw}=5.45$ using equation 5.

(c) Model B inferred source spectra scaled using $\square=0.5$. (c) Model $\mathrm{C}$ inferred source spectra scaled using $[=0.0$. Note the Model $\mathrm{C}$ spectral shapes in (d) do not seem consistent with self-similar scaling. 

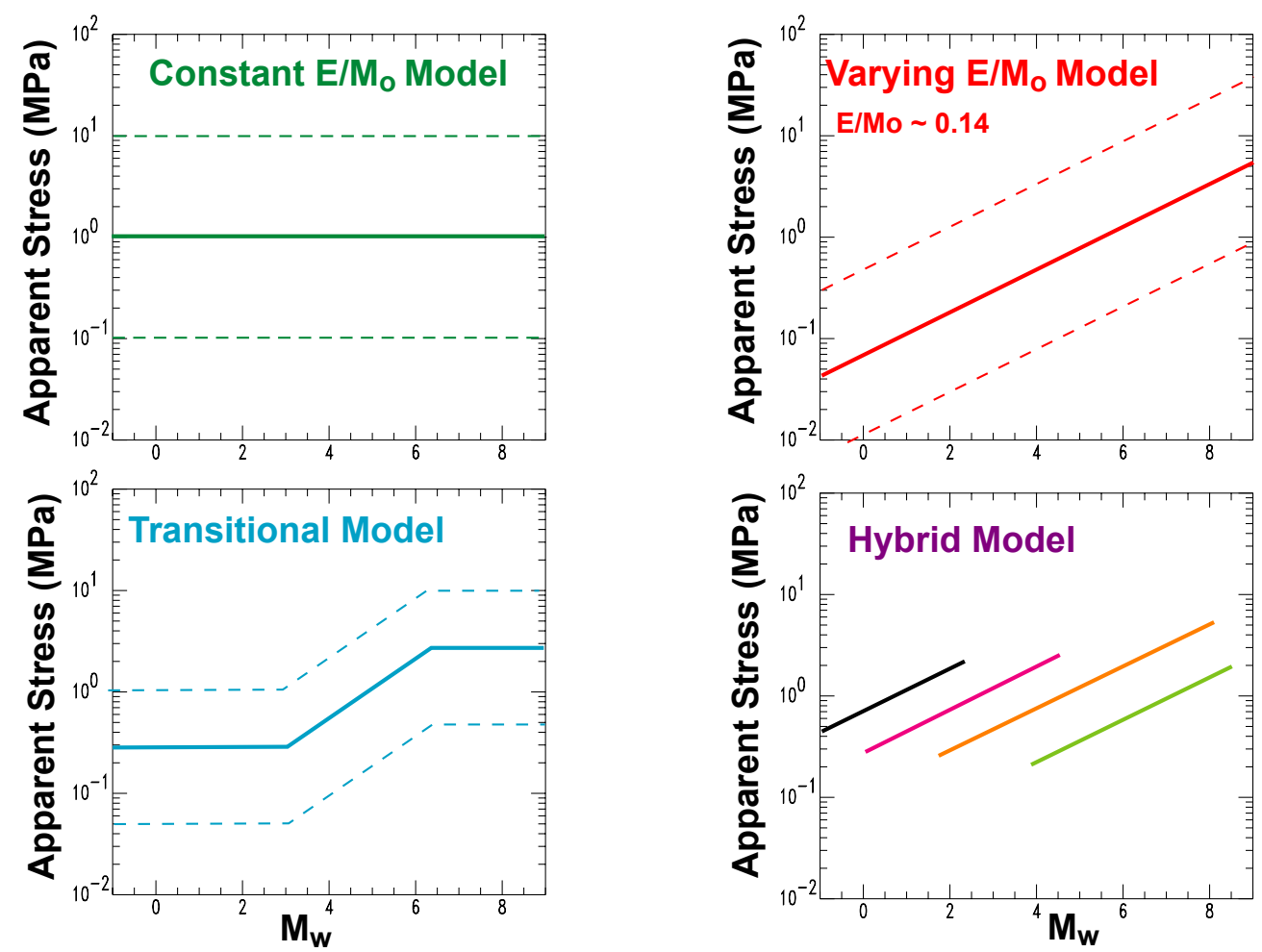

Figure 15. Four different hypothetical models for apparent stress or scaled energy behavior of earthquakes that appear to be consistent with data analyzed to date. (a) A self-similar constant scaled energy model with large variance. (b) A non-self-similar model in which apparent stress increases linearly with moment. (c) A transitional model where small and large earthquakes have constant but different apparent stress levels and intermediate events show a linear increase of apparent stress with moment. (d) A hybrid model in which earthquake sequences within a given region show show linear increases of apparent stress with moment, and each region has a different apparent stress level and maximum earthquake size. 